\title{
The TMEM106B rs1990621 protective variant is also associated with increased neuronal proportion
}

Zeran $\mathrm{Li}^{1,2}$, Fabiana G. Farias ${ }^{1,2}$, Umber Dube ${ }^{1,2}$, Jorge L. Del-Aguila ${ }^{1,2}$, Kathie A.

Mihindukulasuriya ${ }^{1,2}$, Maria Victoria Fernandez ${ }^{1,2}$, Laura Ibanez $z^{1,2}$, John P. Budde ${ }^{1,2}$, Fengxian Wang $^{1,2}$, Allison M. Lake ${ }^{3}$, Yuetiva Deming ${ }^{4}$, James Perez ${ }^{1,2}$, Chengran Yang ${ }^{1,2}$, Joseph

Bradley $^{1,2}$, Richard Davenport ${ }^{1,2}$, Kristy Bergmann ${ }^{1,2}$, Bruno A. Benitez ${ }^{1,2}$, Joseph D.

Dougherty $^{1}$, Oscar Harari ${ }^{1,2}$, Carlos Cruchaga ${ }^{1,2, \Pi}$

1.Washington University School of Medicine, St Louis, MO, USA

2. Hope Center for Neurological Disorders, St Louis, MO, USA

3. Vanderbilt University Medical Scientist Training Program, Nashville, TN, USA

4. University of Wisconsin-Madison, Madison, WI, USA.

$\Pi$ To whom correspondence should be addressed:

Carlos Cruchaga, $\mathrm{PhD}$

Professor

Department of Psychiatry

The Hope Center Program on Protein Aggregation and Neurodegeneration

Washington University, School of Medicine

425 S. Euclid Ave.

BJC Institute of Heath. Box 8134

St. Louis, MO 63110

Tel: 314-286-0546

Fax: 314-362-2244

Email: ccruchaga@wustl.edu 


\section{Abstract}

Background: In previous studies, we observed decreased neuronal and increased astrocyte proportions in $\mathrm{AD}$ cases in parietal brain cortex by using a deconvolution method for bulk RNAseq. These findings suggested that genetic risk factors associated with AD etiology have a specific effect in the cellular composition of $\mathrm{AD}$ brains. The goal of this study is to investigate if there are genetic determinants for brain cell compositions.

Methods: Using cell type composition inferred from transcriptome as a disease status proxy, we performed cell type association analysis to identify novel loci related to cellular population changes in disease cohort. We imputed and merged genotyping data from seven studies in total of 1,669 samples and derived major CNS cell type proportions from cortical RNAseq data. We also inferred RNA transcript integrity number (TIN) to account for RNA quality variances. The model we performed in the analysis was: normalized neuronal proportion $\sim \mathrm{SNP}+$ Age + Gender $+\mathrm{PC} 1+\mathrm{PC} 2+$ median TIN.

Results: A variant rs1990621 located in the TMEM106B gene region was significantly associated with neuronal proportion $\left(p=6.40 \times 10^{-07}\right)$ and replicated in an independent dataset. The association became more significant as we combined both discovery and replication datasets in multi-tissue meta-analysis $\left(\mathrm{p}=9.42 \times 10^{-09}\right)$ and joint analysis $\left(\mathrm{p}=7.66 \times 10^{-10}\right)$. This variant is in high LD with rs1990622 (r2=0.98) which was previously identified as a protective variant in FTD cohorts. Further analyses indicated that this variant is associated with increased neuronal proportion in participants with neurodegenerative disorders, not only in AD cohort but also in cognitive normal elderly cohort. However, this effect was not observed in a younger schizophrenia cohort with a mean age of death $<65$. The second most significant loci for neuron 
proportion was $A P O E$, which suggested that using neuronal proportion as an informative endophenotype could help identify loci associated with neurodegeneration.

Conclusion: This result suggested a common pathway involving TMEM106B shared by aging groups in the present or absence of neurodegenerative pathology may contribute to cognitive preservation and neuronal protection.

\section{Introduction}

Although neuronal loss and synapse dysfunction are the preceding events of cognitive deficits in Alzheimer's disease (AD), neurons do not work or survive by themselves. These delicate organelles require supports through intimate collaborations within themselves and with other cell types ${ }^{1}$. The microenvironment of cellular crosstalk, interaction, balance, and circuits maintained by neurons, astrocytes, microglia, oligodendrocytes, and other vascular cells are essential for the brain to carry out functions and fight against insults.

$\mathrm{AD}$ associated risk factors identified across the genome also point to the involvements of multi-cell types apart from neurons ${ }^{1,2}$. APOE4 is related to lipid metabolism and mostly expressed in astrocyte and microglia ${ }^{3}$. Other lipid metabolism related risk genes are $A B C A 7$ identified in all cell types ${ }^{2,4}, C L U$ in astrocyte and oligodendrocyte precursor cells ${ }^{2,5,6}$, and SORL1 in astrocyte ${ }^{2}$. Research interests in the roles of inflammatory response to toxic stimuli or microbial infection have been escalating recently, and AD risk genes associated with immune response including TREM2 $2^{7-9}, P L C G 2^{9}, A B I 3^{9}, C R 1^{2,6}, C D 33^{2,4}, H L A-D R B 5-H L A-D R B 1^{2}$, and INPP5D $D^{2}$ are mostly expressed in microglia and macrophages. BIN1 expressed in microglia, oligodendrocyte, and neurons ${ }^{2}$, and PICALM expressed in microglia and endothelial cells ${ }^{2,5}$ are associated with endocytosis. 
In a normal functional brain, astrocytes, microglia, and oligodendrocytes provide trophic supports to neurons and various cell type specific functions. Astrocytes confer multiple functions to fulfill neurons' metabolic needs ${ }^{10}$ including but not limited to providing substrates for oxidative phosphorylation ${ }^{11}$, exerting regulation of excitatory CNS neurotransmitter glutamate $^{12,13}$, and serving as bidirectional communication nodes that talk to both neurons and blood vessels and modulate their activities in an arrangement of functional entities named neurovascular units ${ }^{14-16}$. Microglia surveil in the extracellular space and look for pathogens or debris to engulf through phagocytosis. Oligodendrocyte provides insulation to neurons by wrapping around the axons with myelin sheath. However, in an AD diseased brain, these supporting cells may become double-edged swords that play beneficial and/or harmful roles as disease progresses. Amyloid- $\beta$ accumulation and clearance are the central events of the amyloid cascade hypothesis. Both astrocyte and microglia have been involved in response to the toxic stimuli of amyloid plaques. During the early stage, microglia ${ }^{17-19}$ and astrocytes ${ }^{19-21}$ accumulate around plaques to phagocytose or degrade those in a protective manner. However, as disease progresses, the chronic and prolonged activation of microglia and astrocytes will be provoked into a damaging pro-inflammatory state and a vicious circle that exacerbate pathology in a harmful manner. Evidence suggested that increased inflammatory cytokine secretion in microglia, and increased production of complement cascade components, and impaired glutamate regulation (unregulated glutamate activity can cause neuronal excitatory cell death) ${ }^{13}$ may contribute to synaptic loss which ultimately leads to cognitive deficits. Disrupted neuronal plasticity due to myelin loss and dysfunctional neurovascular units further exacerbate the dreadful situation and destroy the harmony of the multi-cell type microenvironment. 
Apart from disturbed homeostatic processes and impaired circuits integrity, cell type composition or proportion is also altered. Brains affected by AD exhibits neuronal loss, oligodendrocyte loss, astrocytosis, and microgliosis. However, the specific effects that pathological mutations and risk variants have on brain cellular composition are often ignored. To investigate the changes of cerebral cortex cell-type population structure and account for the associated confounding effects in downstream analysis, we developed an in-silico deconvolution method to infer cellular composition from RNA-Seq data, which has been documented in our previous publication $^{22}$. In summary, we firstly assembled a reference panel to model the transcriptomic signature of neurons, astrocytes, oligodendrocytes and microglia. The panel was created by analyzing expression data from purified cell lines. We evaluated various digital deconvolution methods and selected the best performing ones for our primary analyses. We tested the digital deconvolution accuracy on induced pluripotent stem cell (iPSC) derived neurons and microglia, and neurons derived from Translating Ribosome Affinity Purification followed by RNA-Seq. Finally, we verified its accuracy with simulated admixture with predefined cellular proportions.

Once the deconvolution approach was optimized, we calculated the cell proportion in AD cases and controls from different brain regions of LOAD and ADAD datasets. We found that neuronal and astrocyte relative proportions differ between healthy and diseased brains, and also differ among $\mathrm{AD}$ cases that carry different genetic risk variants. Brain carriers of pathogenic mutations in APP, PSEN1 or PSEN2 presented lower neuronal and higher astrocytes relative proportions compared to sporadic AD. Similarly, APOE $\varepsilon 4$ carriers also showed decreased neuronal and increased astrocyte relative proportions compared to AD non-carriers. In contrast, carriers of variants in TREM2 risk showed a lower degree of neuronal loss than matched AD 
cases in multiple independent studies. These findings suggest that different genetic risk factors associated with $\mathrm{AD}$ etiology may have gene specific effects in the cellular composition of AD brains.

In a recently published study named PsychENCODE ${ }^{23}$, a very similar deconvolution approach as reported in our previous study ${ }^{22}$ was taken to infer cell type composition from RNASeq data predominantly drawn from psychiatric disorder cohorts. From the cell fractions inferred from bulk RNA-Seq data, they found that cell type composition differences can account for more than $88 \%$ of bulk tissue expression variation observed across the population with a $\pm 4 \%$ variance on a per-subject level. Using cell type compositions as quantitative traits, the authors identified a non-coding variant closed to the FZD9 gene that is associated with both FZD9 gene expression and the proportion of excitatory layer 3 neurons ${ }^{23}$. Interestingly, deletion variants found previously upstream of FZD9 were associated with cell composition changes in Williams syndrome $^{24}$, a developmental disorder exhibits mild to moderate intellectual disabilities with learning deficits and cardiovascular problems. This observation re-emphasized the importance of incorporating cell type composition into RNA-Seq analysis pipeline even in psychiatric disorder cohorts without dramatic changes in cellular composition, not mention the necessity of such practice in neurodegeneration disorders that have significant changes in cell type composition. It also demonstrated the great potential of using relative abundance of specific cell types in identifying novel variants and genes implicated in disease. However, it is unclear if this finding is only applicable to psychiatry-relate traits or it is a more general finding.

In this study, we utilized cell-type proportions inferred from our deconvolution method ${ }^{22}$ to perform cell type QTL analysis in a dataset enriched for AD cases in search for potential new loci that are associated with neurodegeneration disorders. We imputed and merged genotyping or 
whole genome sequencing data from seven studies - five centered on neurodegeneration $(\mathrm{N}=$ $1,125)$, one schizophrenia cohort $(\mathrm{N}=414)$, and GTEx multiple tissue controls $(\mathrm{N}=130)$. From cortical RNA-Seq data, we derived cell fractions of four major CNS cell types, including neuron, astrocyte, microglia, and oligodendrocyte. Using normalized neuronal proportion as quantitative trait, we identified a variant rs 1990621 located in the TMEM106B gene region significantly associated with neuronal proportion variation in all cohorts except schizophrenia subjects. This variant is in high LD with $\mathrm{rs} 1990622\left(\mathrm{r}^{2}=0.98\right)$, which was previously identified as a protective variant in FTD cohorts ${ }^{25}$. Variants in this region have also been found to be associated with AD with TDP-43 pathology ${ }^{26}$, and downregulation of TMEM106B is observed in AD brains ${ }^{27}$. In conclusion, we have identified a variant associated with neuronal proportion with potential protective effect in neurodegeneration disorders.

\section{Methods}

\section{Study participants}

The participants were sourced from seven studies with a total sample size of 1,669

(Table 1). Among those, five studies are mainly focused on neurodegenerative disorders including Alzheimer's disease $(\mathrm{N}=681)$, frontotemporal dementia $(\mathrm{N}=11)$, progressive supranuclear palsy $(\mathrm{N}=82)$, pathological aging $(\mathrm{N}=29)$, Parkinson Disease $(\mathrm{N}=1)$, as well as cognitive normal individuals $(\mathrm{N}=540)$. These samples come from the Mayo, MSSM, Knight ADRC, DIAN, and ROSMAP studies as described in Table 1. To compare with the neurodegenerative disorders, we also included schizophrenia $(\mathrm{N}=210)$ and bipolar disorders $(\mathrm{N}$ =34) participants from the CommonMind study (Table 1). Additionally, two studies, MSSM 
and GTEx, contain multi-tissue data that include some participants contribute more than one tissue (Table 1).

\section{Standard protocol approvals, registrations and patient consents}

The protocol of DIAN and Knight-ADRC studies have been approved by the review board of Washington University in St. Louis. The protocol of Mayo dataset was approved by the Mayo Clinic Institutional Review Board (IRB). All neuropsychological, diagnostic and autopsy protocols of MSSM dataset were approved by the Mount Sinai and JJ Peters VA Medical Center Institutional Review Boards. The religious orders study and the memory and aging project of ROSMAP was approved by the IRB of Rush University Medical Center. The NIH Common Fund's GTEx program protocol was reviewed by Chesapeake Research Review Inc., Roswell Park Cancer Institute's Office of Research Subject Protection, and the institutional review board of the University of Pennsylvania. Within CommonMind consortium, the MSSM sample protocol was approved by Icahn School of Medicine at Mount Sinai IRB; the Pitt sample protocol was approved by the University of Pittsburgh's Committee for the Oversight of Research involving the Dead and IRB for Biomedical Research; the Penn sample protocol was approved by the Committee on Studies Involving Human Beings of the University of Pennsylvania. All participants were recruited with informed consent for research use.

\section{Data collection and generation}

Cortical tissues from various locations of post-mortal brains were collected (Table 2).

RNA was extracted from lysed tissues and prepared into libraries of template molecules ready

for subsequent next-generation sequencing steps. Ribosomal RNAs constitute $80 \%-90 \%$ of total RNAs, which are not the targets of this study. To focus on mRNA quantification usually 
researchers would either remove excessive rRNAs or enrich for mRNAs during RNA-Seq library preparation. In this study, DIAN ${ }^{22}$, Knight $\mathrm{ADRC}^{22}, \mathrm{MSSM}^{28}$, and CommonMind ${ }^{29}$ took a rRNA depletion approach to removed ribosomal RNA from total RNAs to retain a higher mRNA content. Whereas, Mayo ${ }^{30}$, $\operatorname{ROSMAP}^{31,32}$, and $\mathrm{GTEx}^{33,34}$ took a poly-A enrichment approach to enrich mRNAs from total RNAs. Genotype information were also collected and sequenced correspondingly. RNAseq paired with genotype data for each participant were either sequenced at Washington University for DIAN and Knight-ADRC studies or downloaded from public database for all the other studies. Please see Table $\mathbf{2}$ and each study reference(s) for more data collection and generation specifications.

\section{Data QC and preprocessing}

\section{Genetic Data}

Stringent quality control (QC) steps were applied to each genotyping array or sequence data. The minimum call rate for single nucleotide polymorphisms (SNPs) and individuals was 98\% and autosomal SNPs not in Hardy-Weinberg equilibrium ( $\mathrm{p}$-value $<1 \times 10^{-06}$ ) were excluded. X-chromosome SNPs were analyzed to verify gender identification. Unanticipated duplicates and cryptic relatedness (Pihat $\geq 0.25$ ) among samples were tested by pairwise genome-wide estimates of proportion identity-by-descent. EIGENSTRAT ${ }^{35}$ was used to calculate principal components. The 1000 Genomes Project Phase 3 data (October 2014), SHAPEIT v2.r837 $7^{36}$, and IMPUTE2 v2.3.2 $2^{37}$ were used for phasing and imputation. Individual genotypes imputed with probability $<0.90$ were set to missing and imputed genotypes with probability $\geq 0.90$ were analyzed as fully observed. Genotyped and imputed variants with $\mathrm{MAF}<0.02$ or IMPUTE2 information score $<0.30$ were excluded. WGS data quality is censored by filtering 
out reads with sequencing depth DP $<6$ and quality GQ $<20$ followed by similar QC approaches as described above for genotyping data. After the QC, all studies including imputed genotype and WGS data was merged into a binary file using Plink for downstream analysis. PCA and IBD analyses were performed on the merged binary files using Plink to keep European ancestry and unrelated participants (Figure 1 and Figure 2).

\section{Expression Data}

FastQC was applied to RNAseq data to examine various aspects of sequencing quality ${ }^{38}$. Outlier samples with high rRNA contents or low sequencing depth were removed from the pool. The remaining samples were aligned to human GRCh37 primary assembly using Star with 2Pass Basic mode (ver 2.5.4b) ${ }^{39}$. Alignment metrics were ascertained by applying Picard CollectRnaSeqMetrics ${ }^{40}$ including reads bias, coverage, ribosomal contents, coding bases, and etc. Following which, transcript integrity number (TIN) for each transcript was calculated on aligned bam files using RSeQC tin.py ${ }^{41}$ (ver 2.6.5). RNAseq coding gene and transcript expression was quantified using Salmon transcript expression quantification (ver 0.7.2) with GENCODE Homo sapiens GRCh37.75 reference genome ${ }^{42}$.

Four major central nerve system cell type proportions were inferred from RNAseq gene expression quantification output as documented in our previous deconvolution study ${ }^{22}$. To briefly explain the deconvolution process, we firstly assembled a reference panel to model the transcriptomic signature of neurons, astrocytes, oligodendrocytes and microglia from purified single cell tissue sources respectively. Using the reference panel and the method populationspecific expression analysis ${ }^{43}$ (PSEA, also named meanProfile in CellMix implementation ${ }^{44}$ ), we calculated four cell type proportions for each subject bulk RNAseq data. For each brain tissue 
collection site of each study, outlier values for each cell type proportion were removed. Mean values for each cell type of each tissue in each study were subtracted from the deconvolution results to center all the distributions to zero mean (Figure 3). Phenotype information from all studies were merged and unified to the same coding paradigm to enable downstream joint analysis; for example, males are all coded as 1 and females are 2.

\section{Data analysis}

For the discovery phase, ROSMAP dataset was analyzed with linear regression model employed in Plink ${ }^{45}$ using normalized neuronal proportion to run quantitative trait analysis. Age, sex, PC1, PC2, and median TIN were used as covariates to account for potential genetic, phenotypic or technical heterogeneity. TIN is calculated directly from post-sequencing results that captures RNA degradation by measuring mRNA integrity directly ${ }^{41}$. Results were depicted as Manhattan plots using R (ver 3.4.3) qqman package ${ }^{46}$ (ver 0.1.4).

For the replication phase, all the other studies except ROSMAP were combined and prepressed to run meta-tissue QTL analysis because MSSM and GTEx contain samples with multiple cortical tissues. Meta-Tissue software installation and data preprocessing were conducted following a four-step instruction documented in the developer website:

http://genetics.cs.ucla.edu/metatissue/install.html. Meta-tissue ${ }^{47}$ processing pipeline calls two main functions, firstly MetaTissueMM ${ }^{47}$ and then followed by Metasoft $\mathrm{f}^{48}$. MetaTissueMM applies a mixed model to account for the heterogeneity of multiple tissue QTL effects. Metasoft performs the meta-analysis while proving a more accurate random effect $\mathrm{p}$-value for multiple tissue analysis and a m-value based on Bayesian inference to indicate how likely a locus is a QTL in each tissue. Similarly, results were depicted as Manhattan plots and visually examined. 
For the final merging phase, both discovery and replication studies were combined to maximize sample size. Apart from meta-tissue analysis by each tissue of each study, a split by disease status analysis was also performed in the final merging phase. Samples from each tissue of each study were also split into disease categories. Resultant subcategory with less than 20 subjects were removed from the analysis to avoid false results due to too small sample size. Similar data preparation and analysis pipeline were enforced as documented above.

QTL analysis results were uploaded to Fuma (v1.3.3d $)^{49}$ to annotation significant SNPs (p-value $<10^{-06}$ ) with GWAScatalog (e91_r2018-02-06) and ANNOVAR (updated 2017-07-17). Gene-based analysis was also performed by Magma (v1.06) ${ }^{50}$ implemented in Fuma.

\section{Data availability}

Mayo: https://www.synapse.org/\#!Synapse:syn5550404

MSSM: https://www.synapse.org/\#!Synapse:syn3157743

ROSMAP: https://www.synapse.org/\#!Synapse:syn3219045

CommonMind: https://www.synapse.org/\#!Synapse:syn2759792

GTEx: https:/www.ncbi.nlm.nih.gov/projects/gap/cgi-bin/study.cgi?study id=phs000424.v7.p2

Knight-ADRC: https://www.synapse.org/\#!Synapse:syn12181323

According to the data request terms, DIAN data are available upon request: http://dian.wustl.edu

\section{Results}

\section{Study Design}


The ROSMAP study containing 523 subjects will be the discovery dataset, and the other six studies are collapsed into replication dataset with 1,146 subjects. Altogether, we have assembled a set of cortical RNA-Seq data comprised of 1,669 participants predominantly focused on neurodegenerative disorders from seven sources (Figure 4, Table 1). Collectively, Mayo, MSSM, Knight ADRC, and ROSMAP studies contributed 664 sporadic AD cases. Apart from sporadic AD, 15 subjects from DIAN study and 2 from Knight-ADRC also harbor PSEN1, PSEN2, and APP mutations that exhibit familial AD inheritance pattern. Other neurodegenerative disorders, including progressive supranuclear palsy (PSP), pathological aging (PA), frontal temporal dementia (FTD), and Parkinson's Disease (PD), are mainly drawn from Mayo and Knight ADRC datasets. Other psychiatric disorders including schizophrenia and bipolar disorders are contributed by the CommonMind study. Besides, 540 control subjects cleared of cognitive dementia or neuropsychiatric symptoms were also included. MSSM and GTEx also included multiple tissue data, which were collected from multiple regions of the same subjects that allow us to perform region specific comparison within the same cohort.

Discovery analysis was performed in ROSMAP study.-In the replication phase, all the other studies were merged to replicate signals identified from the discovery ROSMAP set. Because GTEx and MSSM contain multiple cortical regions collected from the same subjects, we also applied meta-tissue software ${ }^{47}$ specifically designed for multi-tissue QTL analysis to perform a mixed model analysis with random effects that account for correlated measurements from multi-tissue individuals. To attain the largest available sample size for this study, the discovery and replication sets were merged to perform the merged multi-tissue QTL analysis in a search for additional signals hidden in previously separated discovery or replication analysis due to lack of power. After merged analysis, the cohorts were split into four major disease status 
groups ( $\mathrm{AD}$, control, schizophrenia, other non-AD neurodegenerative disorders) to explore how different disease strata could impact the results.

\section{TMEM106B variants associated with neuronal proportion}

During discovery phase, ROSMAP dataset $(\mathrm{N}=484$ after removing outliers from total number of 523 subjects) was used to perform cell type proportion QTL analysis. Using normalized neuronal proportion as a quantitative trait, the QTL analysis identified more than 10 peaks that passed genome wide suggestive threshold $\left(<1.0 \times 10^{-06}\right.$, Figure 5AB, Table 3).

However, only one signal rs1990621 (chr7: 12283873) were replicated with $\mathrm{p}$-value $=7.41 \times 10^{-04}$ in the replication dataset $(\mathrm{N}=1,052)$ combining all the other datasets except ROSMAP (Figure 5CD). When the discovery and replication datasets were merged to attained a larger sample size $(\mathrm{N}=1,536)$, rs 1990621 major allele $\mathrm{C}$ is negatively associated with neuronal proportion with $\mathrm{p}$ value $=9.42 \times 10^{-09}($ Figure 6AB, Figure 7AC $)$, which means the minor allele $\mathrm{G}$ is associated with increased neuronal proportion in our assembled datasets focusing on neurodegenerative disorders.

Noticeably, in both replication and merged analyses, multi-tissue data were involved that provided additional power but also posed challenges to the analysis, the same issue faced by the GTEx study ${ }^{34,51}$. Compared to a tissue-by-tissue approach, multiple tissues collected from the same subject may help identify QTL by aggregating evidence from multiple tissues, which is similar to a meta-analysis of combining each study. However, one violation of such approach is that the tissues collected from the same subject are presumably highly correlated since they shared the same genetic architecture. Thus, it violates the assumption of independency for carrying out a standard meta-analysis ${ }^{47}$. Another challenge of the multi-tissue QTL is the 
heterogeneity of the effects, which means a variant may have different effects on different tissues. To resolve these issues, we applied the Meta-Tissue analytic pipeline ${ }^{47}$ (http://genetics.cs.ucla.edu/metatissue/) specifically designed for multi-tissue QTL, the same approach that GTEx took to analyze their multi-tissue data. As shown in Figure 7A, Meta-Tissue analysis results of rs 1990621 for the merged analysis were displayed as a forest plot with $95 \%$ confidence interval and p-value labeled for each tissue of each study. Among them, MSSM and GTEx are multi-tissue studies while the others are single-tissue studies. Meta-Tissue used a linear mixed model to capture the multi-tissue correlation within MSSM and GTEx respectively. Regarding the effect heterogeneity, Meta-Tissue calculated a m-value ${ }^{48}$ to predict if a variant has an effect in a tissue. M-value is similar to the posterior probability of association based on the Bayes factor ${ }^{48}$ but with differences specifically designed for detecting whether an effect is present in a study included in a meta-analysis. Figure 7B is a PM-Plot that integrates evidences from both frequentist ( $\mathrm{p}$-value) and Bayesian (m-value) sides to interpret the heterogeneity of multi-tissue QTL effects. Variant rs1990621 in ROSMAP and Mayo studies have m-values greater than 0.9 , are predicted to have an effect and color coded with red. In CMC study, the mvalue is less than 0.1 , so it is predicted to not to have an effect and color coded with blue. All the other studies with m-value between 0.1 and 0.9 are predicted with ambiguous effect and color coded with green. Based on the forest plot and PM-Plot, the variant does have effect heterogeneity across different tissues and studies. In this case, random-effect model will be more suitable to account for effect heterogeneity. Therefore, summary random effect and p-value were reported for the analysis.

Apart from multi-tissue QTL, a single-tissue joint analysis was also performed. In this case, one tissue region was drawn from the multi-tissue data to avoid violating the independency 
assumption. Specifically, BM36 and frontal cortex tissue were selected to represent MSSM and GTEx study respectively. Study sites were coded as dummy variables to account for potential batch effects. In this joint analysis, the variant rs1990621 is also the top hit with p-value $=$ $7.66 \times 10^{-10}$

\section{Neuronal protective effect of TMEM106B variants observed in neurodegenerative disorders and normal aging participants}

To explore the effect in different disease categories, the merged dataset was stratified based on disease: $\mathrm{AD}$, other non-AD neurodegenerative disorders, schizophrenia and control. Signification associations between rs1990621 and neuronal proportion were observed in AD (pvalue $\left.=1.95 \times 10^{-07}\right)$, other non-AD neurodegenerative $\left(p\right.$-value $\left.=8.19 \times 10^{-04}\right)$, and cognitive normal control $\left(\mathrm{p}\right.$-value $\left.=2.94 \times 10^{-02}\right)$ cohorts, but not in schizophrenic cohort $(\mathrm{p}$-value $=$ $9.32 \times 10^{-01}$, Table 4, Figure 7D). The effect of the variant was more prominent in neurodegenerative cohorts and aging controls with mean age of death greater than 65 years old. However, it was absent from younger cohorts such as GTEx controls and CommonMind schizophrenia participants. Thus, this variant seems to be associated with a neuronal protection mechanism shared by any aging process in the present or absence of neuropathology.

\section{Functional annotation}

The variant rs1990621 is located in the TMEM106B gene region where other variants in high LD linkage are also located and labeled in Figure 8A. Although the CADD score and RegulomeDB score for this variant are not remarkably high to suggest any functional consequences (Figure 8BC), this variant is in high LD with $\mathrm{rs} 1990622\left(\mathrm{r}^{2}=0.98\right)$, a TMEM106B variant previous identified to be associated with FTD risk $^{25}$, particularly in granulin precursor 
(GRN) mutation carriers ${ }^{52,53}$. TMEM106B is expressed in neurons and microglia, with highest protein expression detected in the late endosome/lysosome compartments of neurons ${ }^{54-57}$. A nonsynonymous variant rs3173615, which is also in high LD with rs1990621 $\left(r^{2}=0.98\right)$, located in the exon 6 of TMEM106B (the dark blue dot in Figure 8B) produces two protein isoforms (p.T185S) that affect TMEM106B protein level through protein degradation mechanism ${ }^{55,58,59}$.

\section{The impact of other neurodegenerative risk loci on neuronal proportion}

To investigate what other AD or FTD variants might have an effect in neuronal proportion QTL analysis, we extracted results for 38 SNPs examined in two large scale genome wide association studies, AD focused (Lambert et al. ${ }^{2}$ ) and FTD focused (Ferrari et al. ${ }^{60}$ ) studies. Among those, only variants located in TMEM106B and $A P O E$ gene regions passed genome wide significant or suggestive threshold. Both rs1990622 (Figure 9A) and rs2075650 (Figure 9B) were found to be associated with FTD reported in Ferrari et al., which were associated with neuronal proportion in this study (Table 5). The top signals in $A P O E$ region are rs283815, rs 769449 , and rs429358 with p-value $<1.22 \times 10^{-05}$. Note that rs429358 is one of the two SNPs that determine $A P O E$ isoforms. Remember that $A P O E \varepsilon 4$ alleles, coded by rs $429358(\mathrm{C})$ and rs 7412(C), confers the largest effect for AD risk. We observed that the $\mathrm{C}$ allele of rs 429358 was associated with decreased neuronal proportion, but no association observed between rs 7412 and neuronal proportion.

In a gene-based analysis of our neuronal proportion QTL, TMEM106B (p-value = $2.96 \times 10^{-08}$ ) is the only gene that passed genome-wide significant threshold followed by $A P O E$ $\left(\mathrm{p}\right.$-value $\left.=3.2 \times 10^{-05}\right)$, the most important gene for sporadic AD risk (Figure 6CD). Previous GWAS for AD risk performed with the International Genomics of Alzheimer's Project (IGAP) 
data stratified by $A P O E$ genotype showed that AD risk is significantly influenced by the interaction between $A P O E$ and $T M E M 106 B^{61}$. Together with our observation of cellular composition QTL, these results suggest a potential interaction of TMEM106B and APOE may play a role in affecting $\mathrm{AD}$ risk/vulnerability and cellular composition balance between neurons and astrocytes, and the endosome and lysosome compartments might be the location that the interaction takes place.

\section{Discussion}

The common variant rs1990622 in TMEM106B was first identified to be associated with FTD with TDP-43 inclusions ${ }^{25}$. Hyper-phosphorylated and ubiquitinated TDP-43 is the major pathological protein for FTD and $\mathrm{ALS}^{62}$, which is also present in a broader range of neurodegenerative disorders, including $\mathrm{AD}^{63}$, Lewy body disease ${ }^{64}$, and hippocampal sclerosis ${ }^{63}$. Recent study also suggested distinct TDP-43 types present in non-FTD brains, typical TDP-43 $\alpha$ type and newly characterized $\beta$-type ${ }^{65}$. TDP-43 $\alpha$-type is the typical form conventionally observed in temporal, frontal and brainstem regions. TDP-43 $\beta$-type is characterized by its close adjacency to neurofibrillary tangles, which is predominantly observed in limbic system, including amygdala, entorhinal cortex, and subiculum of the hippocampus. These findings suggested that pathologic TDP-43 protein that closely associated with TMEM106B variants might be the common pathologic substrate linking these neurodegenerative disorders. Multiple lines of evidence have merged and shown that protective variants in TMEM106B are associated with attenuated cognitive deficits or better cognitive performance in $\operatorname{ALS}^{66}$, hippocampal sclerosis $^{67}$, presymptomatic FTD $^{68}$, and aging groups with various neuropathological burden ${ }^{69}$ or in the absence of known brain disease ${ }^{70}$. Our study identified a protective variant rs1990621 of 
$T M E M 106 B$ is associated with increased neuronal proportion in participants with neurodegenerative disorders and normal aging in non-demented controls. However, this effect is not observed in a younger schizophrenia cohort with a mean age of death less than 65 years old. This result suggested a common pathway involving TMEM106B shared by aging groups in the present or absence of neurodegenerative pathology that may contribute to cognitive preservation and neuronal protection.

Our study has demonstrated that a protective variant rs1990621 identified in TMEM106B gene region may exert neuronal protection function in aging groups. A protein coding variant rs3173615 in high LD with rs $1990621\left(\mathrm{r}^{2}=0.98\right)$ produces two protein isoforms (p.T185S). The S185 allele is protective and the protein carrying this amino acid is degraded faster than the risk variant T185. Thus, the risk allele of this coding variant leads to increased TMEM106B protein level ${ }^{55,58,59}$. TMEM106B overexpression results in enlarged lysosomes and lysosomal dysfunction $^{55,71}$. It has also been shown that TMEM106B may interact with PGRN (the precursor protein for granulin) in lysosome ${ }^{59}$. Although rs3173615 is not included in our genomic data, it is in complete linkage disequilibrium with rs 1990621 and rs1990622. It is worth pointing out that the minor allele of rs 1990622, which has a protective effect in FTD, is in-phase with the minor allele of rs1990621, which is associated with increased neuronal proportion in our analysis. Despite the fact that our dataset is focused on neurodegeneration, we only have 11 verified FTD cases suggesting that TMEM106B might have a general neuronal protection role in neurodegeneration apart from FTD.

This observation suggested that a potential involvement of TMEM106B in the endosome/lysosome pathway may play a role in neurodegenerative disorder risk or vulnerability. Neuronal survival requires continuous lysosomal turnover of cellular contents through 
endocytosis and autophagy ${ }^{72}$. Impaired lysosomal function reduces lysosomal degradative efficiency, which leads to abnormal build-up of toxic components in the cell. Impaired lysosomal system has been found to be associated with a broad range of neurodegenerative disorders, including $\mathrm{AD}^{73}$, Parkinson disease ${ }^{74-76}$, Huntington disease ${ }^{77,78}, \mathrm{FTD}^{79}, \mathrm{ALS}^{77}$, Niemann-Pick disease type $\mathrm{C}^{80,81}$, Creutzfeldt-Jakob disease ${ }^{82}$, Charcot-Marie Tooth disease type $2 \mathrm{~B}^{83}$, Neuronal ceroid lipofuscinoses (Batten disease) ${ }^{84,85}$, autosomal dominant hereditary spastic paraplegia $^{86}$, Chediak-Higashi syndrome ${ }^{87}$, inclusion body myositis ${ }^{88}$, and osteopetrosis ${ }^{89}$. Considering the extensive involvements of lysosomal/endosomal compartments in neurodegenerative disorders, it has been proposed that a long and chronic process of abnormal metabolic changes during aging has led to the accumulation of toxic materials ${ }^{72}$. When lifespan increases especially in the sporadic forms of neurodegenerative disorders, failures to degrade these waste products break the proteostasis and the balance maintained by the multicellular interactions, and trigger subsequent chain reactions that lead to neuronal death and outbreaks of various neurodegenerative disorders due to different genetic susceptibilities and other disease etiologies. Although each neurodegenerative disorder has its own characteristic proteopathy, the boundaries of protein pathology distribution are never clear-cut across different disorders. In fact, copathology or nonspecific pathology of proteopathy have been observed in most autopsies of neurodegenerative disorders, such as TDP-43 discussed above, Lewy body, $\alpha$-synuclein ${ }^{90}$, and etc. Our observation of lysosomal gene TMEM106B associated with neuronal proportion in aging cohorts suggests that the lysosomal pathway might be involved in the common mechanism underlying a broad range of neurodegenerative disorders or aging process in general that contribute to neuronal cell death. 
Our study has demonstrated the great potential of using cell type composition as quantitative traits to identify QTLs associated with the changes in cell fractions. This approach is more powerful for disorders that involve considerably changes in cellular composition, for example, neurodegenerative disorders, and normal conditions during developmental or aging processes. The development of recent single cell studies will greatly increase the resolution in advancing our knowledge of cellular population changes. More detailed fine mapping of cellular composition from single cell studies together with machine learning algorithms, bulk RNA-Seq deconvolution will be more accurately capturing cellular fraction changes in the samples, such as different types of neurons or different states of astrocytes or microglia. Regarding scalability, this single cell powered bulk deconvolution approach is preferable for carrying out such cell type composition QTL analysis, because due to the high cost of performing single cell studies, bulk RNA-Seq is more financially feasible to scale up, and with larger sample sizes more hidden signals will be unrevealed with increased statistical power.

To conclude, we have identified a protective variant rs 1990621 in TMEM106B associated with increased neuronal proportion through bulk RNA-Seq deconvolution and cell type proportion QTL analysis. This observation also replicated previous findings of the protective variant rs 1990622 in FTD risk, which is in high LD with rs $19990621^{25}$. Besides, we also observed the $\mathrm{C}$ allele of rs429358 (codetermine $A P O E \& 4$ isoform with rs $7412 \mathrm{C}$ allele) associated with decreased neuronal proportion as it was hypothesized. It suggested potential involvements of both $A P O E$ and TMEM106B in neuronal protection mechanisms underlying neurodegenerative and normal aging processes, and supported previous observation of interactions between these two genes ${ }^{61}$ in $\mathrm{AD}$ cohort. We speculate that $T M E M 106 B$ related lysosomal changes might be involved in the common pathway underlying neuronal death and 
astrocytosis in neurodegenerative disorders and normal aging cohorts. With larger sample size and higher deconvolution resolution, this approach will reveal more biologically relevant and novel loci associated with changes in cellular composition that are important for understanding both disease etiology and healthy aging. 


\section{References}

1 Henstridge, C. M., Hyman, B. T. \& Spires-Jones, T. L. Beyond the neuron-cellular interactions early in Alzheimer disease pathogenesis. Nature reviews. Neuroscience 20, 94-108, doi:10.1038/s41583-018-0113-1 (2019).

2 Lambert, J. C. et al. Meta-analysis of 74,046 individuals identifies 11 new susceptibility loci for Alzheimer's disease. Nat Genet 45, 1452-1458, doi:10.1038/ng.2802 (2013).

3 Corder, E. H. et al. Gene dose of apolipoprotein E type 4 allele and the risk of Alzheimer's disease in late onset families. Science 261, 921-923 (1993).

4 Hollingworth, P. et al. Common variants at ABCA7, MS4A6A/MS4A4E, EPHA1, CD33 and CD2AP are associated with Alzheimer's disease. Nat Genet 43, 429-435, doi:10.1038/ng.803 (2011).

5 Harold, D. et al. Genome-wide association study identifies variants at CLU and PICALM associated with Alzheimer's disease. Nat Genet 41, 1088-1093, doi:10.1038/ng.440 (2009).

6 Lambert, J. C. et al. Genome-wide association study identifies variants at CLU and CR1 associated with Alzheimer's disease. Nat Genet 41, 1094-1099, doi:ng.439 [pii] 10.1038/ng.439 (2009).

7 Guerreiro, R. et al. TREM2 variants in Alzheimer's disease. N Engl J Med 368, 117-127, doi:10.1056/NEJMoa1211851 (2013).

8 Jonsson, T. et al. Variant of TREM2 Associated with the Risk of Alzheimer's Disease. $N$ Engl J Med, doi:10.1056/NEJMoa1211103 (2012).

9 Sims, R. et al. Rare coding variants in PLCG2, ABI3, and TREM2 implicate microglialmediated innate immunity in Alzheimer's disease. Nat Genet 49, 1373-1384, doi:10.1038/ng.3916 (2017).

10 Stobart, J. L. \& Anderson, C. M. Multifunctional role of astrocytes as gatekeepers of neuronal energy supply. Frontiers in cellular neuroscience 7, 38, doi:10.3389/fncel.2013.00038 (2013).

11 Pellerin, L. et al. Evidence supporting the existence of an activity-dependent astrocyteneuron lactate shuttle. Developmental neuroscience 20, 291-299, doi:10.1159/000017324 (1998).

12 Fonnum, F. Glutamate: a neurotransmitter in mammalian brain. J Neurochem 42, 1-11 (1984).

13 Dong, X. X., Wang, Y.\& Qin, Z. H. Molecular mechanisms of excitotoxicity and their relevance to pathogenesis of neurodegenerative diseases. Acta pharmacologica Sinica $\mathbf{3 0}$, 379-387, doi:10.1038/aps.2009.24 (2009).

14 Tsacopoulos, M. \& Magistretti, P. J. Metabolic coupling between glia and neurons. $J$ Neurosci 16, 877-885 (1996).

15 Simard, M., Arcuino, G., Takano, T., Liu, Q. S. \& Nedergaard, M. Signaling at the gliovascular interface. J Neurosci 23, 9254-9262 (2003).

16 Rouach, N., Koulakoff, A., Abudara, V., Willecke, K. \& Giaume, C. Astroglial metabolic networks sustain hippocampal synaptic transmission. Science 322, 1551-1555, doi:10.1126/science.1164022 (2008).

17 Heneka, M. T. et al. Neuroinflammation in Alzheimer's disease. Lancet Neurol 14, 388405, doi:10.1016/s1474-4422(15)70016-5 (2015). 
18 Hickman, S., Izzy, S., Sen, P., Morsett, L. \& El Khoury, J. Microglia in neurodegeneration. Nat Neurosci 21, 1359-1369, doi:10.1038/s41593-018-0242-x (2018).

19 Heppner, F. L., Ransohoff, R. M. \& Becher, B. Immune attack: the role of inflammation in Alzheimer disease. Nature reviews. Neuroscience 16, 358-372, doi:10.1038/nrn3880 (2015).

20 Pekny, M. et al. Astrocytes: a central element in neurological diseases. Acta Neuropathol 131, 323-345, doi:10.1007/s00401-015-1513-1 (2016).

21 Rodriguez-Arellano, J. J., Parpura, V., Zorec, R. \& Verkhratsky, A. Astrocytes in physiological aging and Alzheimer's disease. Neuroscience 323, 170-182, doi:10.1016/j.neuroscience.2015.01.007 (2016).

$22 \mathrm{Li}, \mathrm{Z}$. et al. Genetic variants associated with Alzheimer's disease confer different cerebral cortex cell-type population structure. Genome Med 10, 43, doi:10.1186/s13073-0180551-4 (2018).

23 Wang, D. et al. Comprehensive functional genomic resource and integrative model for the human brain. Science 362, doi:10.1126/science.aat8464 (2018).

24 Chailangkarn, T. et al. A human neurodevelopmental model for Williams syndrome. Nature 536, 338-343, doi:10.1038/nature19067 (2016).

25 Van Deerlin, V. M. et al. Common variants at $7 \mathrm{p} 21$ are associated with frontotemporal lobar degeneration with TDP-43 inclusions. Nat Genet 42, 234-239, doi:10.1038/ng.536 (2010).

26 Rutherford, N. J. et al. TMEM106B risk variant is implicated in the pathologic presentation of Alzheimer disease. Neurology 79, 717-718, doi:10.1212/WNL.0b013e318264e3ac (2012).

27 Satoh, J. et al. TMEM106B expression is reduced in Alzheimer's disease brains. Alzheimers Res Ther 6, 17, doi:10.1186/alzrt247 (2014).

28 Wang, M. et al. The Mount Sinai cohort of large-scale genomic, transcriptomic and proteomic data in Alzheimer's disease. Sci Data 5, 180185, doi:10.1038/sdata.2018.185 (2018).

29 Fromer, M. et al. Gene expression elucidates functional impact of polygenic risk for schizophrenia. Nat Neurosci 19, 1442-1453, doi:10.1038/nn.4399 (2016).

30 Allen, M. et al. Human whole genome genotype and transcriptome data for Alzheimer's and other neurodegenerative diseases. Sci Data 3, 160089, doi:10.1038/sdata.2016.89 (2016).

31 Bennett, D. A., Schneider, J. A., Arvanitakis, Z. \& Wilson, R. S. Overview and findings from the religious orders study. Curr Alzheimer Res 9, 628-645 (2012).

32 Bennett, D. A. et al. Overview and findings from the rush Memory and Aging Project. Curr Alzheimer Res 9, 646-663 (2012).

33 The Genotype-Tissue Expression (GTEx) project. Nat Genet 45, 580-585, doi:10.1038/ng.2653 (2013).

34 Battle, A., Brown, C. D., Engelhardt, B. E. \& Montgomery, S. B. Genetic effects on gene expression across human tissues. Nature 550, 204-213, doi:10.1038/nature24277 (2017).

35 Price, A. L. et al. Principal components analysis corrects for stratification in genomewide association studies. Nat Genet 38, 904-909 (2006).

36 Delaneau, O. \& Marchini, J. Integrating sequence and array data to create an improved 1000 Genomes Project haplotype reference panel. Nature communications 5, 3934, doi:10.1038/ncomms4934 (2014). 
37 Howie, B., Fuchsberger, C., Stephens, M., Marchini, J. \& Abecasis, G. R. Fast and accurate genotype imputation in genome-wide association studies through pre-phasing. Nat Genet 44, 955-959, doi:10.1038/ng.2354 (2012).

38 FastQC: a quality control tool for high throughput sequence data (2010).

39 Dobin, A. et al. STAR: ultrafast universal RNA-seq aligner. Bioinformatics 29, 15-21, doi:10.1093/bioinformatics/bts635 (2013).

40 Broad Institute The Picard Pipeline, $<$ http://broadinstitute.github.io/picard/> (

41 Wang, L. et al. Measure transcript integrity using RNA-seq data. BMC Bioinformatics 17, 58, doi:10.1186/s12859-016-0922-z (2016).

42 Patro, R., Duggal, G., Love, M. I., Irizarry, R. A. \& Kingsford, C. Salmon provides fast and bias-aware quantification of transcript expression. Nat Methods 14, 417-419, doi:10.1038/nmeth.4197 (2017).

43 Kuhn, A., Thu, D., Waldvogel, H. J., Faull, R. L. \& Luthi-Carter, R. Population-specific expression analysis (PSEA) reveals molecular changes in diseased brain. Nat Methods $\mathbf{8}$, 945-947, doi:10.1038/nmeth.1710 (2011).

44 Gaujoux, R. \& Seoighe, C. CellMix: a comprehensive toolbox for gene expression deconvolution. Bioinformatics 29, 2211-2212, doi:10.1093/bioinformatics/btt351 (2013).

45 Purcell, S. et al. PLINK: a tool set for whole-genome association and population-based linkage analyses. Am J Hum Genet 81, 559-575 (2007).

46 Turner, S. D. qqman: an R package for visualizing GWAS results using Q-Q and manhattan plots. bioRxiv, 005165, doi:10.1101/005165 (2014).

47 Sul, J. H., Han, B., Ye, C., Choi, T. \& Eskin, E. Effectively identifying eQTLs from multiple tissues by combining mixed model and meta-analytic approaches. PLoS Genet 9 , e1003491, doi:10.1371/journal.pgen.1003491 (2013).

48 Han, B. \& Eskin, E. Interpreting meta-analyses of genome-wide association studies. PLoS Genet 8, e1002555, doi:10.1371/journal.pgen.1002555 (2012).

49 Watanabe, K., Taskesen, E., van Bochoven, A. \& Posthuma, D. Functional mapping and annotation of genetic associations with FUMA. Nature communications 8, 1826, doi:10.1038/s41467-017-01261-5 (2017).

50 de Leeuw, C. A., Mooij, J. M., Heskes, T. \& Posthuma, D. MAGMA: generalized geneset analysis of GWAS data. Plos Comput Biol 11, e1004219, doi:10.1371/journal.pcbi.1004219 (2015).

51 Consortium, G. T. Human genomics. The Genotype-Tissue Expression (GTEx) pilot analysis: multitissue gene regulation in humans. Science 348, 648-660, doi:10.1126/science.1262110 (2015).

52 Cruchaga, C. et al. Association of TMEM106B gene polymorphism with age at onset in granulin mutation carriers and plasma granulin protein levels. Arch Neurol 68, 581-586, doi:10.1001/archneurol.2010.350 (2011).

53 Finch, N. et al. TMEM106B regulates progranulin levels and the penetrance of FTLD in GRN mutation carriers. Neurology 76, 467-474, doi:10.1212/WNL.0b013e31820a0e3b (2011).

54 Lang, C. M. et al. Membrane orientation and subcellular localization of transmembrane protein 106B (TMEM106B), a major risk factor for frontotemporal lobar degeneration. $J$ Biol Chem 287, 19355-19365, doi:10.1074/jbc.M112.365098 (2012). 
55 Brady, O. A., Zheng, Y., Murphy, K., Huang, M. \& Hu, F. The frontotemporal lobar degeneration risk factor, TMEM106B, regulates lysosomal morphology and function. Hum Mol Genet 22, 685-695, doi:10.1093/hmg/dds475 (2013).

56 Schwenk, B. M. et al. The FTLD risk factor TMEM106B and MAP6 control dendritic trafficking of lysosomes. Embo j 33, 450-467, doi:10.1002/embj.201385857 (2014).

57 Stagi, M., Klein, Z. A., Gould, T. J., Bewersdorf, J. \& Strittmatter, S. M. Lysosome size, motility and stress response regulated by fronto-temporal dementia modifier

TMEM106B. Molecular and cellular neurosciences 61, 226-240, doi:10.1016/j.men.2014.07.006 (2014).

58 Chen-Plotkin, A. S. et al. TMEM106B, the risk gene for frontotemporal dementia, is regulated by the microRNA-132/212 cluster and affects progranulin pathways. $J$

Neurosci 32, 11213-11227, doi:10.1523/jneurosci.0521-12.2012 (2012).

59 Nicholson, A. M. et al. TMEM106B p.T185S regulates TMEM106B protein levels: implications for frontotemporal dementia. J Neurochem 126, 781-791, doi:10.1111/jnc.12329 (2013).

60 Ferrari, R. et al. Frontotemporal dementia and its subtypes: a genome-wide association study. Lancet Neurol 13, 686-699, doi:10.1016/s1474-4422(14)70065-1 (2014).

61 Jun, G. et al. A novel Alzheimer disease locus located near the gene encoding tau protein. Mol Psychiatry 21, 108-117, doi:10.1038/mp.2015.23 (2016).

62 Neumann, M. et al. Ubiquitinated TDP-43 in frontotemporal lobar degeneration and amyotrophic lateral sclerosis. Science 314, 130-133, doi:10.1126/science.1134108 (2006).

63 Amador-Ortiz, C. et al. TDP-43 immunoreactivity in hippocampal sclerosis and Alzheimer's disease. Ann Neurol 61, 435-445, doi:10.1002/ana.21154 (2007).

64 Nakashima-Yasuda, H. et al. Co-morbidity of TDP-43 proteinopathy in Lewy body related diseases. Acta Neuropathol 114, 221-229, doi:10.1007/s00401-007-0261-2 (2007).

65 Josephs, K. A. et al. Pathological, imaging and genetic characteristics support the existence of distinct TDP-43 types in non-FTLD brains. Acta Neuropathol 137, 227-238, doi:10.1007/s00401-018-1951-7 (2019).

66 Vass, R. et al. Risk genotypes at TMEM106B are associated with cognitive impairment in amyotrophic lateral sclerosis. Acta Neuropathol 121, 373-380, doi:10.1007/s00401010-0782-y (2011).

67 Murray, M. E. et al. Differential clinicopathologic and genetic features of late-onset amnestic dementias. Acta Neuropathol 128, 411-421, doi:10.1007/s00401-014-1302-2 (2014).

68 Premi, E. et al. Cognitive reserve and TMEM106B genotype modulate brain damage in presymptomatic frontotemporal dementia: a GENFI study. Brain 140, 1784-1791, doi:10.1093/brain/awx103 (2017).

69 White, C. C. et al. Identification of genes associated with dissociation of cognitive performance and neuropathological burden: Multistep analysis of genetic, epigenetic, and transcriptional data. PLoS medicine 14, e1002287, doi:10.1371/journal.pmed.1002287 (2017).

70 Rhinn, H. \& Abeliovich, A. Differential Aging Analysis in Human Cerebral Cortex Identifies Variants in TMEM106B and GRN that Regulate Aging Phenotypes. Cell Syst 4, 404-415.e405, doi:10.1016/j.cels.2017.02.009 (2017). 
71 Zhou, X., Sun, L., Brady, O. A., Murphy, K. A. \& Hu, F. Elevated TMEM106B levels exaggerate lipofuscin accumulation and lysosomal dysfunction in aged mice with progranulin deficiency. Acta Neuropathologica Communications 5, 9 , doi:10.1186/s40478-017-0412-1 (2017).

72 Nixon, R. A., Yang, D. S. \& Lee, J. H. Neurodegenerative lysosomal disorders: a continuum from development to late age. Autophagy 4, 590-599 (2008).

73 Nixon, R. A. Endosome function and dysfunction in Alzheimer's disease and other neurodegenerative diseases. Neurobiol Aging 26, 373-382, doi:10.1016/j.neurobiolaging.2004.09.018 (2005).

74 Anglade, P. et al. Apoptosis and autophagy in nigral neurons of patients with Parkinson's disease. Histology and histopathology 12, 25-31 (1997).

75 Sarkar, S., Davies, J. E., Huang, Z., Tunnacliffe, A. \& Rubinsztein, D. C. Trehalose, a novel mTOR-independent autophagy enhancer, accelerates the clearance of mutant huntingtin and alpha-synuclein. J Biol Chem 282, 5641-5652, doi:10.1074/jbc.M609532200 (2007).

76 Webb, J. L., Ravikumar, B., Atkins, J., Skepper, J. N. \& Rubinsztein, D. C. AlphaSynuclein is degraded by both autophagy and the proteasome. J Biol Chem 278, 2500925013, doi:10.1074/jbc.M300227200 (2003).

77 Filimonenko, M. et al. Functional multivesicular bodies are required for autophagic clearance of protein aggregates associated with neurodegenerative disease. J Cell Biol 179, 485-500, doi:10.1083/jcb.200702115 (2007).

78 Vonsattel, J. P. \& DiFiglia, M. Huntington disease. J Neuropathol Exp Neurol 57, 369384 (1998).

79 Lee, J. A., Beigneux, A., Ahmad, S. T., Young, S. G. \& Gao, F. B. ESCRT-III dysfunction causes autophagosome accumulation and neurodegeneration. Curr Biol 17, 1561-1567, doi:10.1016/j.cub.2007.07.029 (2007).

80 Ko, D. C. et al. Cell-autonomous death of cerebellar purkinje neurons with autophagy in Niemann-Pick type C disease. PLoS Genet 1, 81-95, doi:10.1371/journal.pgen.0010007 (2005).

81 Pacheco, C. D., Kunkel, R. \& Lieberman, A. P. Autophagy in Niemann-Pick C disease is dependent upon Beclin-1 and responsive to lipid trafficking defects. Hum Mol Genet 16, 1495-1503, doi:10.1093/hmg/ddm100 (2007).

82 Laszlo, L. et al. Lysosomes as key organelles in the pathogenesis of prion encephalopathies. J Pathol 166, 333-341, doi:10.1002/path.1711660404 (1992).

83 Shirk, A. J., Anderson, S. K., Hashemi, S. H., Chance, P. F. \& Bennett, C. L. SIMPLE interacts with NEDD4 and TSG101: evidence for a role in lysosomal sorting and implications for Charcot-Marie-Tooth disease. J Neurosci Res 82, 43-50, doi:10.1002/jnr.20628 (2005).

84 Koike, M. et al. Participation of autophagy in storage of lysosomes in neurons from mouse models of neuronal ceroid-lipofuscinoses (Batten disease). Am J Pathol 167, 1713-1728, doi:10.1016/s0002-9440(10)61253-9 (2005).

85 Koike, M. et al. Involvement of two different cell death pathways in retinal atrophy of cathepsin D-deficient mice. Molecular and cellular neurosciences 22, 146-161 (2003).

86 Reid, E. et al. The hereditary spastic paraplegia protein spastin interacts with the ESCRTIII complex-associated endosomal protein CHMP1B. Hum Mol Genet 14, 19-38, doi:10.1093/hmg/ddi003 (2005). 
87 Kypri, E., Schmauch, C., Maniak, M. \& De Lozanne, A. The BEACH protein LvsB is localized on lysosomes and postlysosomes and limits their fusion with early endosomes. Traffic (Copenhagen, Denmark) 8, 774-783, doi:10.1111/j.1600-0854.2007.00567.x (2007).

88 Askanas, V. \& Engel, W. K. Inclusion-body myositis: newest concepts of pathogenesis and relation to aging and Alzheimer disease. J Neuropathol Exp Neurol 60, 1-14 (2001).

89 Jentsch, T. J. Chloride and the endosomal-lysosomal pathway: emerging roles of CLC chloride transporters. The Journal of physiology 578, 633-640, doi:10.1113/jphysiol.2006.124719 (2007).

90 Espay, A. J. et al. Revisiting protein aggregation as pathogenic in sporadic Parkinson and Alzheimer diseases. Neurology 92, 329-337, doi:10.1212/wnl.0000000000006926 (2019). 
Table 1 Demographic information for cohorts included in the study. AD: Alzheimer's Disease; FTD: frontal temporal dementia; PSP: progressive supranuclear palsy; PA: pathological aging; PD: Parkinson's Disease; SCZ: schizophrenia; BP: bipolar disease; OTH: other unknown dementia or no diagnosis information.

\begin{tabular}{|c|c|c|c|c|c|c|c|c|c|c|c|c|c|c|c|}
\hline & $\mathbf{N}$ & Region & Age & $\%$ Male & RIN & TIN & Control & AD & FTD & PSP & PA & PD & SCZ & BP & OTH \\
\hline \multicolumn{16}{|c|}{ Discovery } \\
\hline ROSMAP & 523 & 1 & $86.6 \pm 4.59$ & 35.4 & $7.07 \pm 0.99$ & $73.2 \pm 5.13$ & 114 & 338 & 0 & 0 & 0 & 0 & 0 & 0 & 71 \\
\hline \multicolumn{16}{|c|}{ Replication } \\
\hline Mayo & 260 & 1 & $80.4 \pm 8.37$ & 48.1 & $8.16 \pm 0.903$ & $77.4 \pm 5.94$ & 69 & 80 & 0 & 82 & 29 & 0 & 0 & 0 & 0 \\
\hline MSSM & 219 & 4 & $84 \pm 7.32$ & 35.6 & $6.42 \pm 1.77$ & $76.4 \pm 2.52$ & 49 & 170 & 0 & 0 & 0 & 0 & 0 & 0 & 0 \\
\hline Knight ADRC & 108 & 1 & $83.1 \pm 12$ & 42.6 & $6.44 \pm 1.2$ & $79.4 \pm 1.91$ & 13 & 77 & 11 & 0 & 0 & 1 & 0 & 0 & 6 \\
\hline DIAN & 15 & 1 & $50.9 \pm 7.08$ & 73.3 & $5.55 \pm 1.09$ & $78.9 \pm 0.99$ & 0 & 15 & 0 & 0 & 0 & 0 & 0 & 0 & 0 \\
\hline GTEx & 130 & 3 & $58.2 \pm 9.91$ & 67.7 & $6.92 \pm 0.846$ & $73.8 \pm 2.97$ & 125 & 1 & 0 & 0 & 0 & 0 & 0 & 0 & 4 \\
\hline CommonMind & 414 & 1 & $64.6 \pm 18$ & 62.3 & $7.67 \pm 0.899$ & $50 \pm 7.21$ & 170 & 0 & 0 & 0 & 0 & 0 & 210 & 34 & 0 \\
\hline Replication Total & 1,146 & & & & & & 426 & 343 & 11 & 82 & 29 & 1 & 210 & 34 & 10 \\
\hline Merged Total & 1,669 & & & & & & 540 & 681 & 11 & 82 & 29 & 1 & 210 & 34 & 81 \\
\hline
\end{tabular}


Table 2 General information of seven studies evolved in the analysis. TCX: temporal cortex; PAR: parietal cortex; CTX: cortex; FCX: frontal cortex; DLPFC: dorsal lateral prefrontal cortex. BM9: dorsal lateral prefrontal cortex; BM10: Anterior prefrontal cortex; BM22: superior temporal gyrus; BM24: ventral anterior cingulate cortex; BM36: parahippocampal gyrus; BM44: inferior frontal gyrus. Mean coverage unit is million.

\begin{tabular}{|c|c|c|c|c|c|c|c|c|}
\hline Dataset & Brain Region & Library Type & Read Length & $\begin{array}{c}\text { mRNA } \\
\text { Enrichment } \\
\end{array}$ & Sequencer & Mean Coverage & $\begin{array}{c}\text { DNA } \\
\text { type }\end{array}$ & Reference \\
\hline \multicolumn{9}{|c|}{ Discovery } \\
\hline ROSMAP & DLPFC & Paired end & 101 & ploy-A selection & $\begin{array}{c}\text { HiSeq } \\
2000\end{array}$ & $99.2 \pm 29.29$ & WGS & $\begin{array}{c}\text { Bennett } \\
\text { 2012; } \\
\text { Bennett } \\
2012 \\
\end{array}$ \\
\hline \multicolumn{9}{|c|}{ Replication } \\
\hline Mayo & $\mathrm{TCX}$ & Paired end & 101 & ploy-A selection & $\begin{array}{c}\text { HiSeq } \\
2000\end{array}$ & $158.31 \pm 34.04$ & Genotype & Allen 2016 \\
\hline MSSM & $\begin{array}{l}\text { BM10 BM22 } \\
\text { BM36 BM44 }\end{array}$ & Single end & 100 & rRNA depletion & $\begin{array}{c}\text { HiSeq } \\
2500\end{array}$ & $35.96 \pm 10.04$ & WGS & Wang 2018 \\
\hline $\begin{array}{l}\text { Knight- } \\
\text { ADRC }\end{array}$ & PAR & Paired end & 150 & rRNA depletion & $\begin{array}{c}\text { HiSeq } \\
4000\end{array}$ & $137.87 \pm 21.81$ & Genotype & Li 2018 \\
\hline DIAN & PAR & Paired end & 150 & rRNA depletion & $\begin{array}{c}\text { HiSeq } \\
4000\end{array}$ & $149.82 \pm 19.68$ & Genotype & Li 2018 \\
\hline GTEx & $\begin{array}{c}\text { BM24 CTX } \\
\text { FCX }\end{array}$ & Paired end & 76 & ploy-A selection & $\begin{array}{c}\text { HiSeq } \\
2000\end{array}$ & $48.28 \pm 13.2$ & WGS & $\begin{array}{l}\text { GTEx 2013; } \\
\text { Battle } 2017\end{array}$ \\
\hline $\begin{array}{l}\text { Common } \\
\text { Mind }\end{array}$ & BM9 & Paired end & 100 & rRNA depletion & $\begin{array}{c}\text { HiSeq } \\
2500\end{array}$ & $86 \pm 21.12$ & Genotype & $\begin{array}{c}\text { Fromer } \\
2016\end{array}$ \\
\hline
\end{tabular}


Table 3 rs1990621 (chr7:12283873) major allele C is significantly associated with decreased neuronal proportions. Therefore, G allele $(\mathrm{MAF}=0.4658)$ is significantly associated with increased neuronal proportions.

\begin{tabular}{llccccc}
\hline Dataset & Brain Region & Ref Allele & Sample Size & Beta & SE & P-value \\
\hline Discovery & DLPFC & C & 484 & -0.3 & 0.06 & $6.40 \times 10^{-07}$ \\
Replication & Multiple & C & 1,052 & -0.13 & 0.04 & $7.41 \times 10^{-04}$ \\
Merged meta-tissue & Multiple & C & 1,536 & -0.16 & 0.05 & $9.42 \times 10^{-09}$ \\
\hline
\end{tabular}


Table 4 rs1990621 (chr7:12283873) major allele C is significantly associated with decreased neuronal proportions in AD, Control, and other non-AD neurodegenerative disorders. SCZ: schizophrenia; other: other non-AD neurodegenerative disorders, including progressive supranuclear palsy and pathological aging. BM9: dorsal lateral prefrontal cortex. TCX: temporal cortex.

\begin{tabular}{llccccc}
\hline Disease & Brain Region & Ref Allele & Sample Size & Beta & SE & P-value \\
\hline AD & Multiple & C & 639 & -0.26 & 0.07 & $1.95 \times 10^{-07}$ \\
Control & Multiple & C & 476 & -0.14 & 0.06 & $2.94 \times 10^{-02}$ \\
SCZ & BM9 & C & 189 & -0.01 & 0.09 & $9.32 \times 10^{-01}$ \\
Other & TCX & C & 103 & -0.45 & 0.14 & $8.19 \times 10^{-04}$ \\
\hline
\end{tabular}


Table 5 Neuronal proportion cQTL p-values were reported for variants previously identified in AD risk (by Lambert et al.) and FTD risk (by Ferrari et al.) studies.

\begin{tabular}{|c|c|c|c|c|c|}
\hline SNP & CHR & BP & cQTL p-value & AD risk p-value & FTD risk p-value \\
\hline rs6656401 & 1 & $207,692,049$ & $5.59 \times 10^{-02}$ & $5.7 \times 10^{-24}$ & - \\
\hline rs35349669 & 2 & $234,068,476$ & $9.80 \times 10^{-01}$ & $3.2 \times 10^{-08}$ & - \\
\hline rs190982 & 5 & $88,223,420$ & $5.88 \times 10^{-01}$ & $3.2 \times 10^{-08}$ & - \\
\hline rs1980493 & 6 & $32,363,215$ & $7.83 \times 10^{-01}$ & - & $1 \cdot 57 \times 10^{-08}$ \\
\hline rs3129871 & 6 & $32,406,342$ & $3.72 \times 10^{-01}$ & - & $3 \cdot 43 \times 10^{-01}$ \\
\hline rs3129882 & 6 & $32,409,530$ & $1.04 \times 10^{-01}$ & - & $3 \cdot 36 \times 10^{-02}$ \\
\hline rs9268856 & 6 & $32,429,719$ & $8.12 \times 10^{-01}$ & - & $5 \cdot 51 \times 10^{-09}$ \\
\hline rs9268877 & 6 & $32,431,147$ & $1.91 \times 10^{-01}$ & - & $1 \cdot 05 \times 10^{-08}$ \\
\hline rs9271192 & 6 & $32,578,530$ & $4.31 \times 10^{-01}$ & $2.9 \times 10^{-12}$ & - \\
\hline rs10948363 & 6 & $47,487,762$ & $8.62 \times 10^{-01}$ & $5.2 \times 10^{-11}$ & - \\
\hline rs1020004 & 7 & $12,255,778$ & $1.35 \times 10^{-03}$ & - & $4 \cdot 59 \times 10^{-01}$ \\
\hline rs6966915 & 7 & $12,265,988$ & $1.24 \times 10^{-08}$ & - & $1 \cdot 21 \times 10^{-01}$ \\
\hline rs1990622 & 7 & $12,283,787$ & $1.44 \times 10^{-08}$ & - & $7 \cdot 88 \times 10^{-02}$ \\
\hline rs2718058 & 7 & $37,841,534$ & $9.99 \times 10^{-01}$ & $4.8 \times 10^{-09}$ & - \\
\hline rs1476679 & 7 & $100,004,446$ & $7.71 \times 10^{-01}$ & $5.6 \times 10^{-10}$ & - \\
\hline rs11771145 & 7 & $143,110,762$ & $9.67 \times 10^{-01}$ & $1.1 \times 10^{-13}$ & - \\
\hline rs28834970 & 8 & $27,195,121$ & $6.38 \times 10^{-02}$ & $7.4 \times 10^{-14}$ & - \\
\hline rs3849942 & 9 & $27,543,281$ & $8.94 \times 10^{-01}$ & - & $4 \cdot 38 \times 10^{-04}$ \\
\hline rs10838725 & 11 & $47,557,871$ & $8.60 \times 10^{-02}$ & $1.1 \times 10^{-08}$ & - \\
\hline rs10792832 & 11 & $85,867,875$ & $4.50 \times 10^{-01}$ & $9.3 \times 10^{-26}$ & - \\
\hline rs302668 & 11 & $87,876,911$ & $4.57 \times 10^{-02}$ & - & $2 \cdot 44 \times 10^{-07}$ \\
\hline rs11218343 & 11 & $121,435,587$ & $1.35 \times 10^{-01}$ & $9.7 \times 10^{-15}$ & - \\
\hline rs17125944 & 14 & $53,400,629$ & $1.71 \times 10^{-01}$ & $7.9 \times 10^{-09}$ & - \\
\hline rs10498633 & 14 & $92,926,952$ & $8.32 \times 10^{-01}$ & $5.5 \times 10^{-09}$ & - \\
\hline rs242557 & 17 & $44,019,712$ & $8.89 \times 10^{-01}$ & - & $4 \cdot 82 \times 10^{-03}$ \\
\hline rs8070723 & 17 & $44,081,064$ & $7.48 \times 10^{-01}$ & - & $2 \cdot 80 \times 10^{-04}$ \\
\hline rs4147929 & 19 & $1,063,443$ & $4.78 \times 10^{-01}$ & $1.1 \times 10^{-15}$ & - \\
\hline rs2075650 & 19 & $45,395,619$ & $2.11 \times 10^{-04}$ & - & $8 \cdot 81 \times 10^{-07}$ \\
\hline rs3865444 & 19 & $51,727,962$ & $2.02 \times 10^{-01}$ & $3.0 \times 10^{-06}$ & - \\
\hline rs7274581 & 20 & $55,018,260$ & $3.01 \times 10^{-01}$ & $2.5 \times 10^{-08}$ & - \\
\hline
\end{tabular}




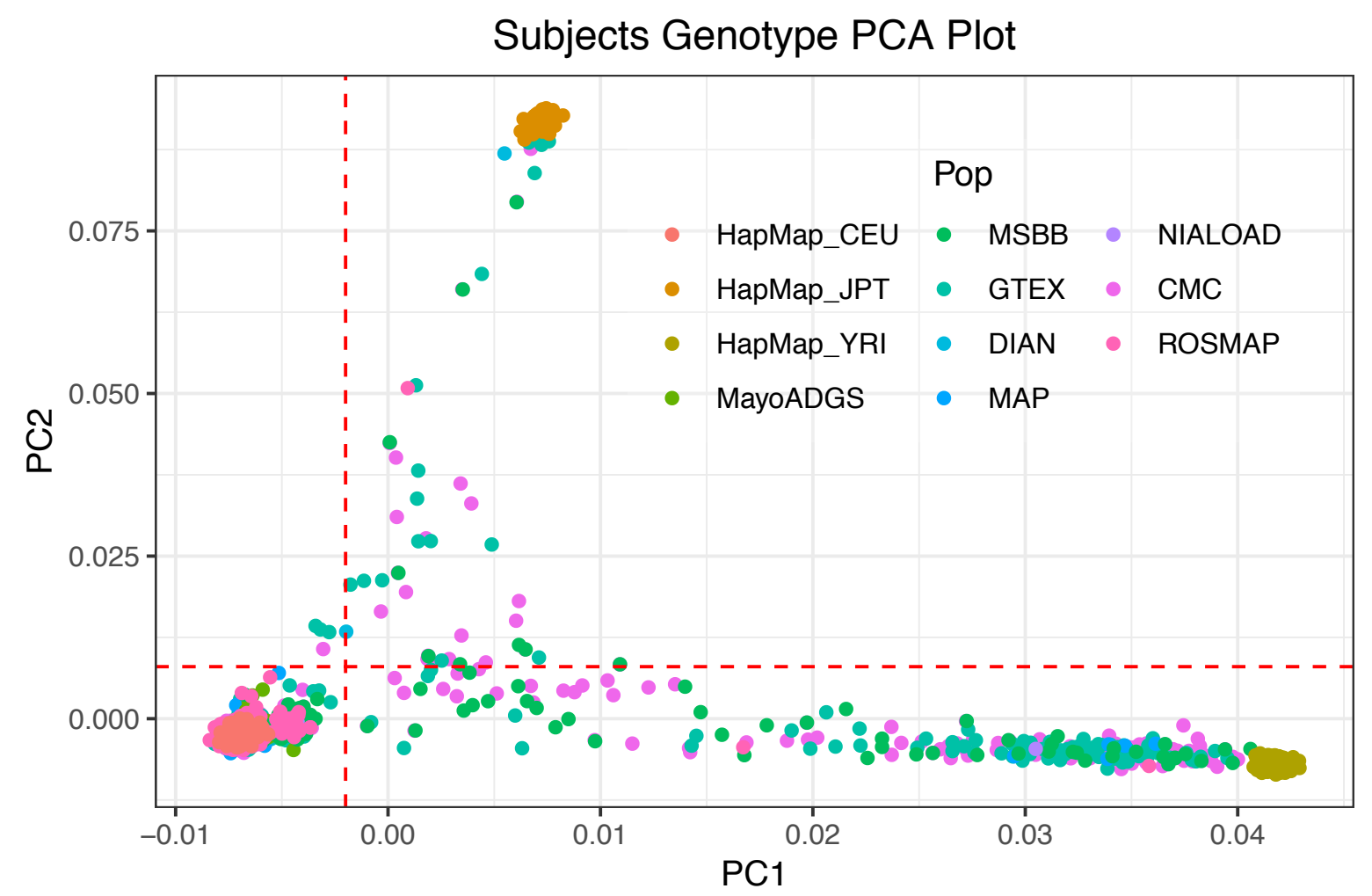

Figure 1 Genomic PCA analysis. Genotype data PCA analysis was performed to select European ancestry subjects with PC $1<-0.002$ and PC $2<0.008$ with red dotted cut-off lines. HapMap_CEU: HapMap Utah residents with Northern and Western European ancestry; HapMap_JPT: HapMap Japanese in Tokyo, Japan; HapMap_YRI: HapMap Yoruba in Ibadan, Nigeria; MayoADGS: Mayo Clinic study participants; MSBB: MSSM study participants; GTEX: GTEx study participants; DIAN: DIAN study participants; MAP: Knight-ADRC participants; NIALOAD: Knight-ADRC participants; CMC: CommonMind participants; ROSMAP: ROSMAP participants. 


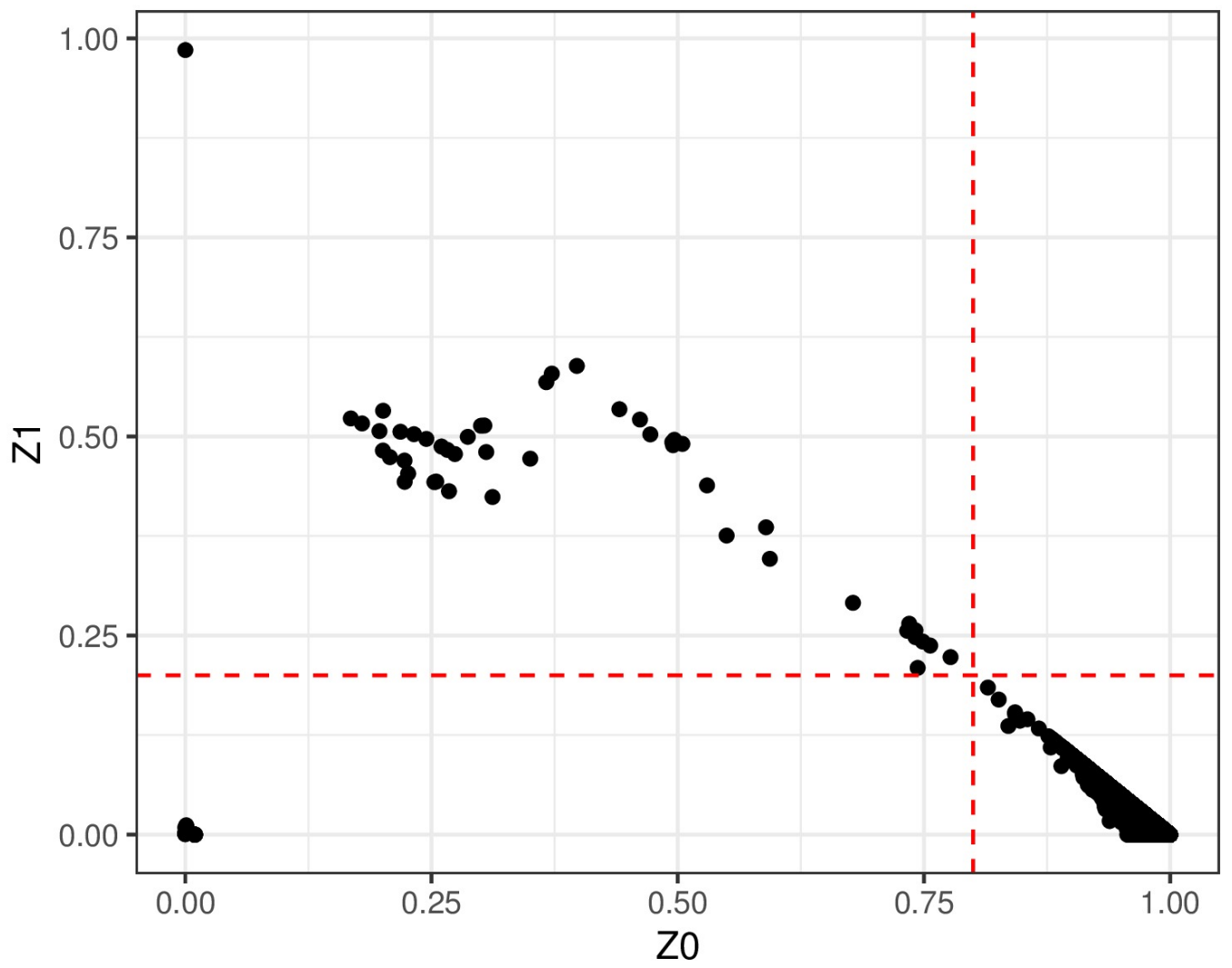

Figure 2 Genomic IBD analysis. IBD analysis was performed to select unrelated subjects with $\mathrm{Z} 0>0.8$ and $\mathrm{Z} 1<0.2$ with red dotted cut-off lines. When there are related individuals, one individual will be dropped from the related pair. 

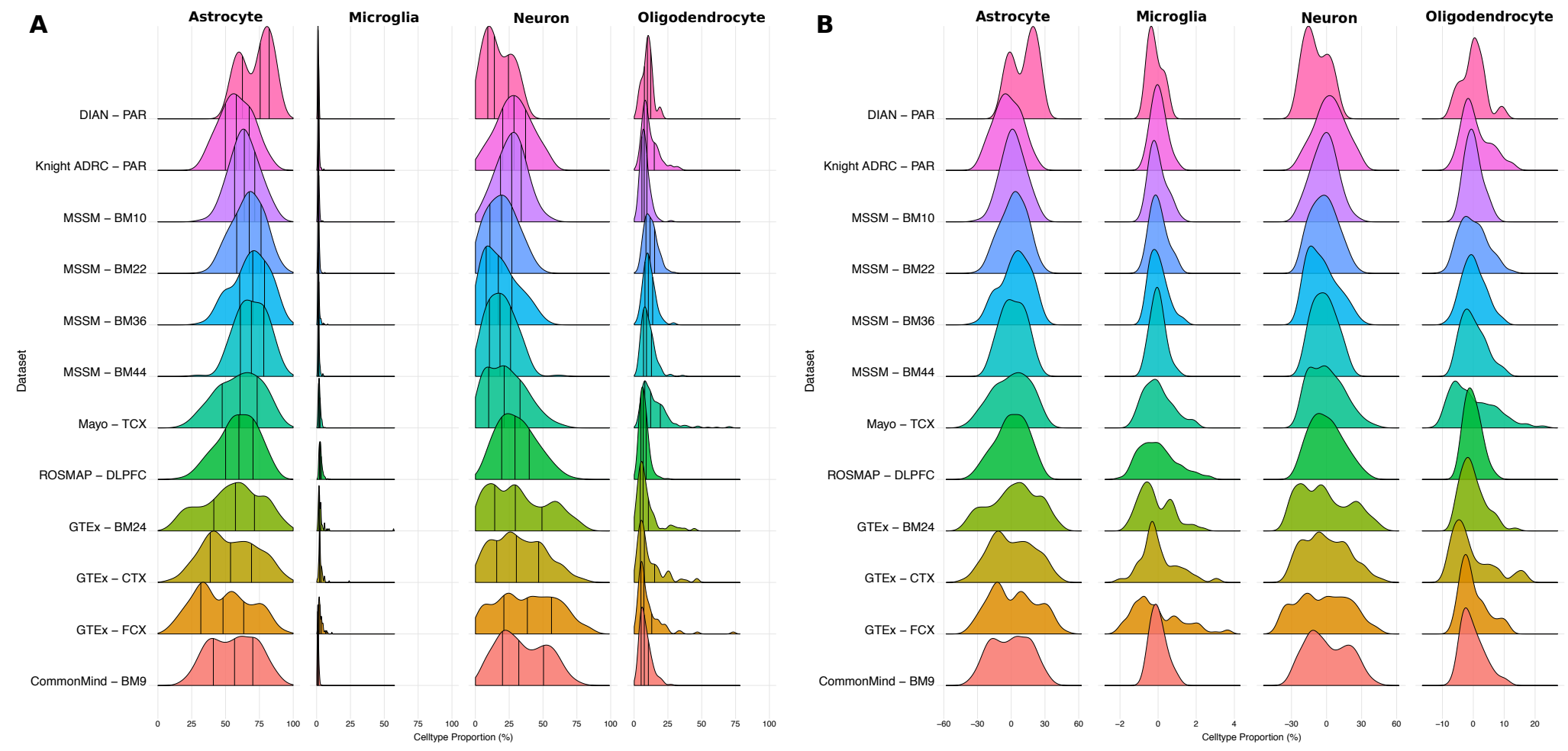

Figure 3 Cell proportion distribution. Major CNS cell type proportions derived from RNAseq datasets with each row representing each tissue of each study A) raw cell type proportions inferred from the data with vertical bars indicating quantiles within each tissue and each cell type. B) cell type proportions were normalized by subtracting the mean from each tissue deconvolution result after removing outliers. 


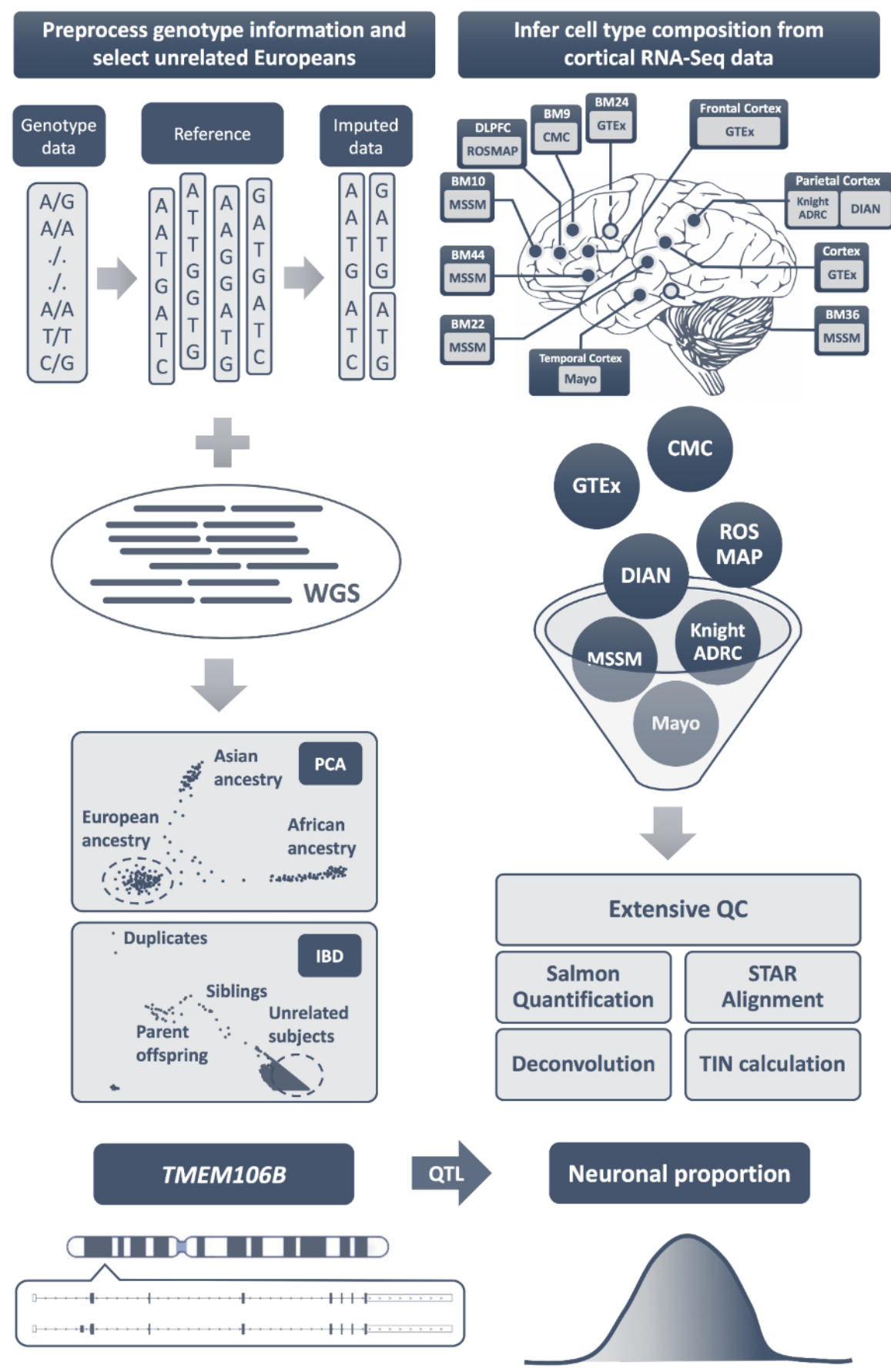

Figure 4 Study Design. RNA-Seq and paired genotype or WGS data were accessed and preprocessed for downstream analysis. Genotype data was censored based on our quality control criteria and imputed as needed. WGS and imputed genotype were merged and followed by PCA and IBD procedures to select unrelated European ancestry subjects. RNA-Seq data was quality checked with FastQC and aligned to human GRCh37 primary assembly with Star, from which TIN was inferred with RSeQC to account for RNA integrity variances that we later incorporated into the analysis. Gene expression were quantified from unaligned RNASeq with psedo-aligner Salmon for deconvolution procedure. Cell type composition comprised of four major CNS cell type proportions were inferred by performing deconvolution procedure on gene expression quantification results. Using cell type proportions as quantitative traits, we identified loci in TMEMI06B gene region associated with neuronal proportion in our assembled dataset. 
A

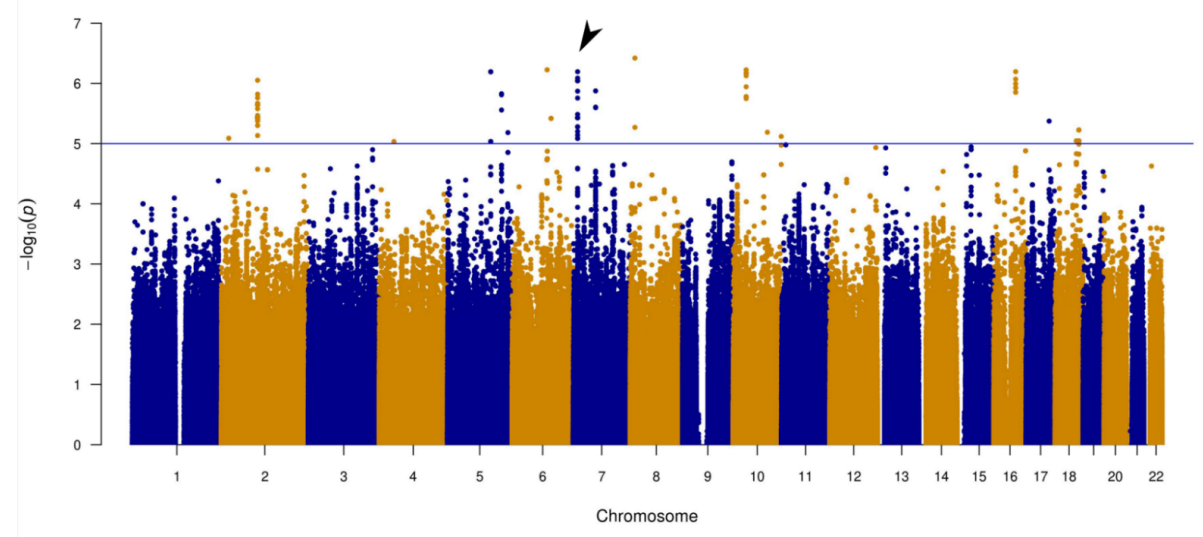

C

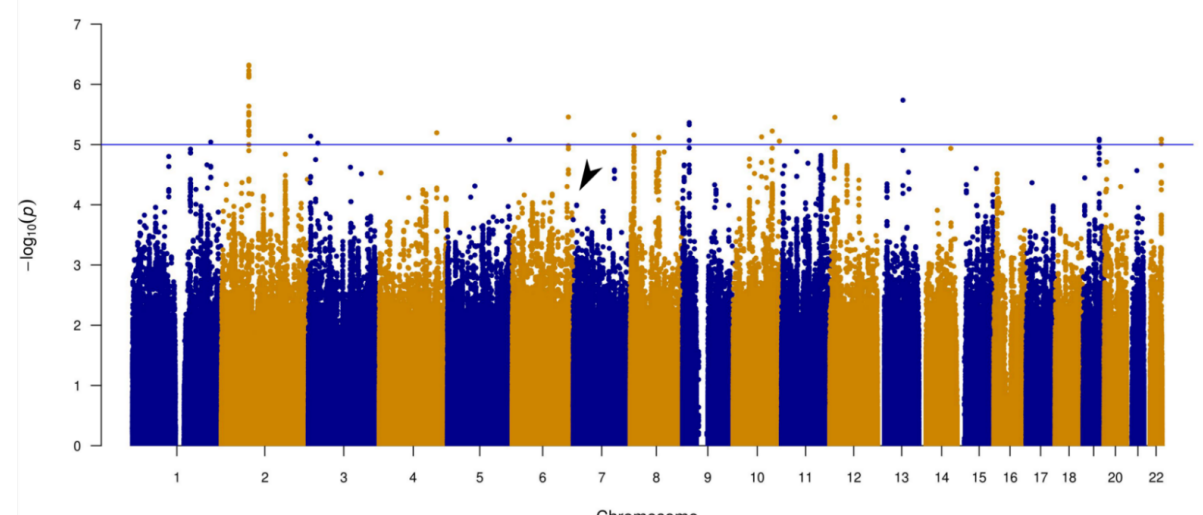

B

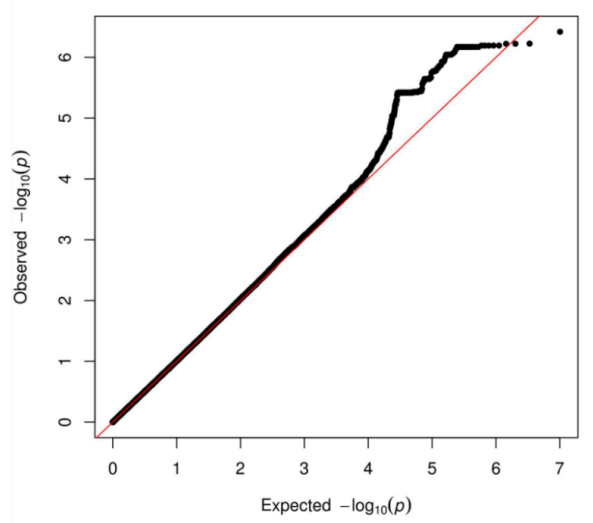

D

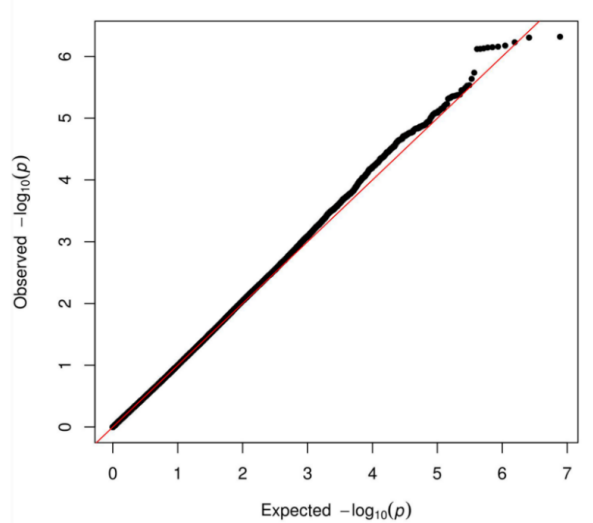

Figure 5 Discovery and replication phases Manhattan and QQ plots. Loci located in chromosome 7 were associated with neuronal proportion in ROSMAP discovery dataset and replicated in replication dataset. A) Discovery set Manhattan plot showed seven peaks associated with neuronal proportion at suggestive threshold. The peak located in chromosome 7 was labeled, which is for rs1990621 with p-value $=6.4 \times 10^{-07}$. B) QQ plot of the discovery phase analysis. C) Replication set Manhattan plot showed that the peak located in chromosome 7 replicated the signal identified during discovery phase with $p$-value $=7.41 \times 10^{-04}$. D) QQ plot of the replication phase analysis. 

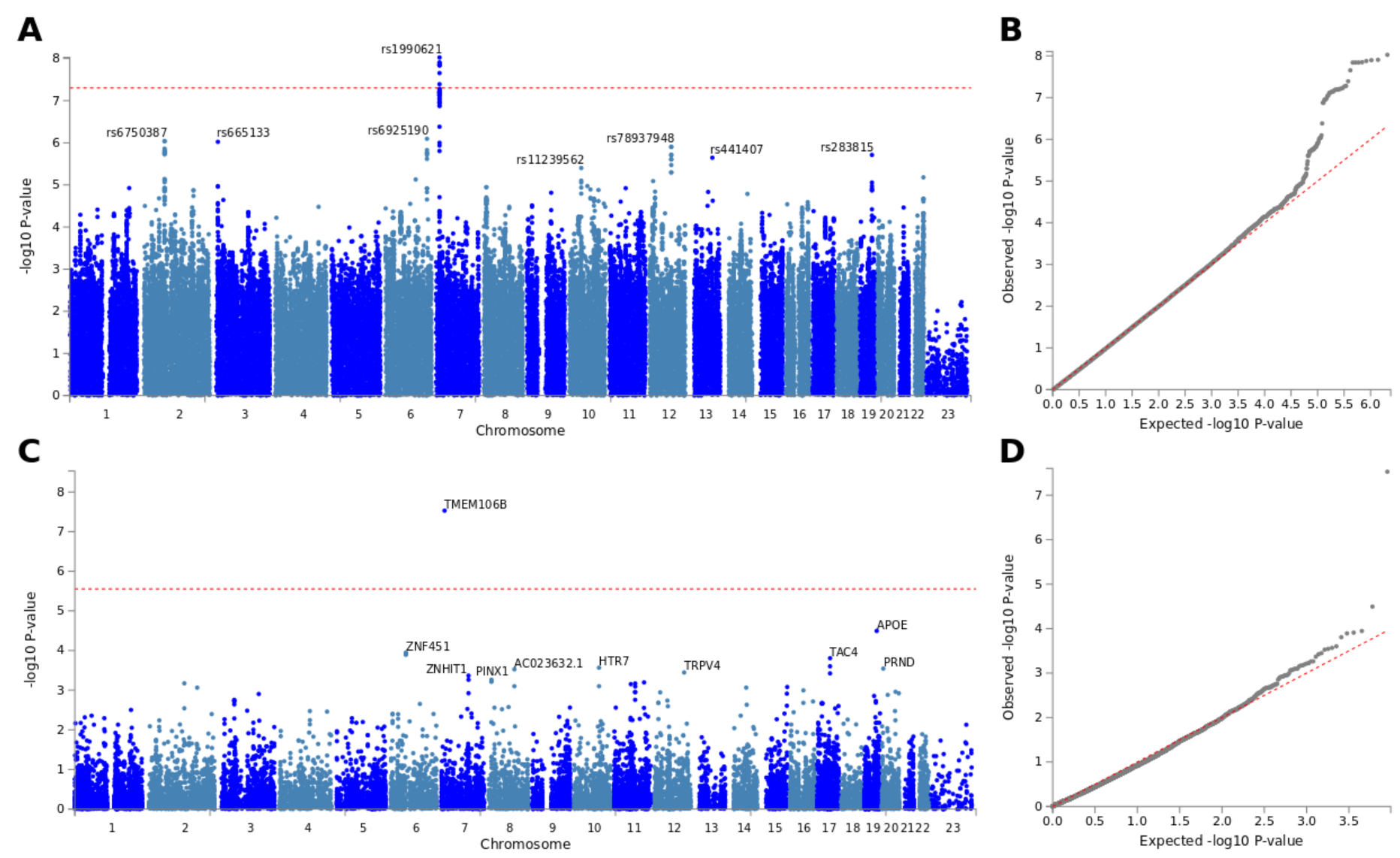

Figure 6 Merged SNP-based and gene-based analysis. rs1990621 located in chromosome 7 TMEM106B gene region was significantly associated with neuronal proportion in cortical RNAseq dataset. A) Manhattan plot showed SNP-based genome-wide significant hit located in chromosome 7 with other suggestive SNP hits labeled. B) QQ plot of the SNP-based analysis. C) Manhattan plot showed gene-based genome-wide significant hit located in chromosome 7 with other suggestive gene hits labeled. D) QQ plot of the gene-based analysis. 
A

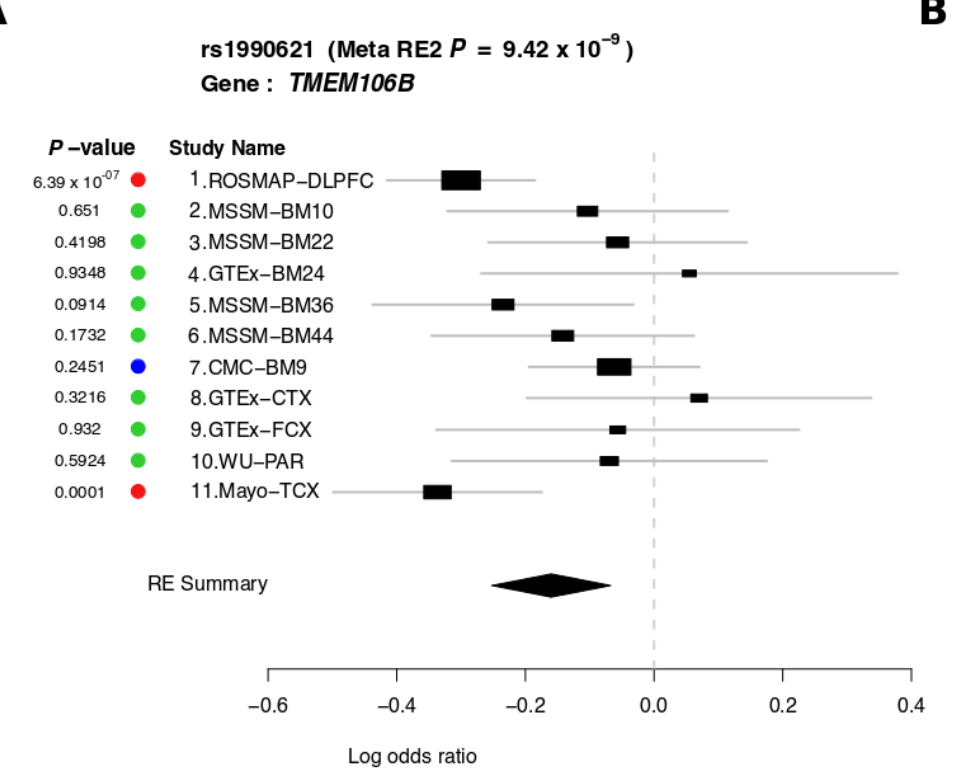

C

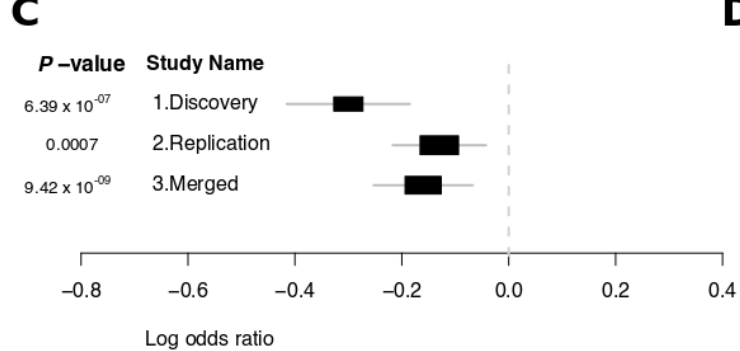

B

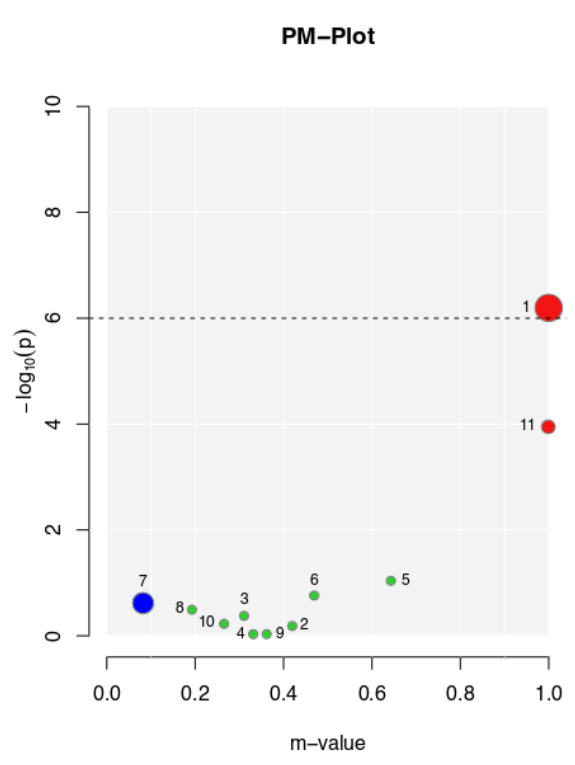

D

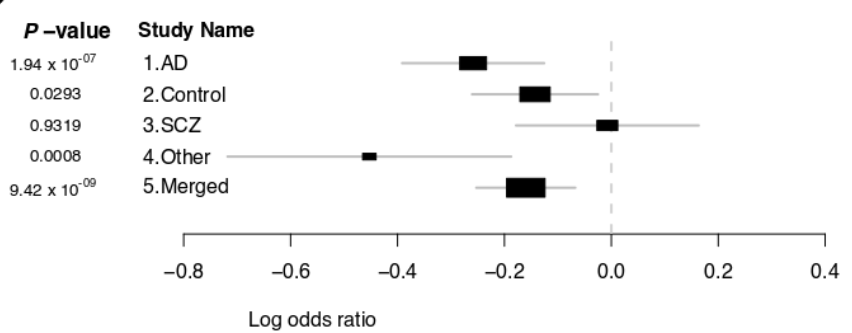

Figure 7 Meta-Tissue analysis results of rs1990621. A) Forest plot showed p-value and confidence interval for rs1990621 for each tissue site of each dataset that included in the Meta-Tissue analysis. Summary random effect was depicted at the bottom as RE Summary. B) PM-Plot of rs 1990621 while combining both p-value (y axis) and $\mathrm{m}$-value ( $\mathrm{x}$ axis). Red dot indicates that the variant is predicted to have an effect in that particular dataset, blue dot means that the variant is predicted to not have an effect, and green dot represents ambiguous prediction. C) Forest plot p-value and confidence interval for rs 1990621 for discovery, replication, and merged datasets. D) Forest plot p-value and confidence interval for rs1990621 when splitting the merged dataset into four main disease categories. 
A

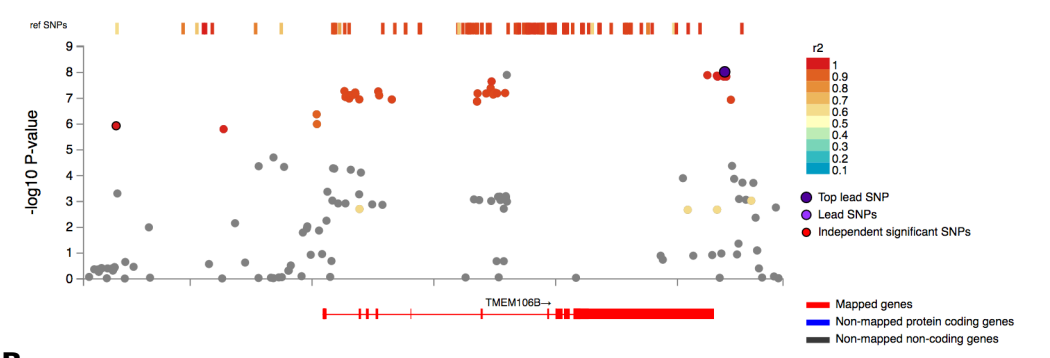

B

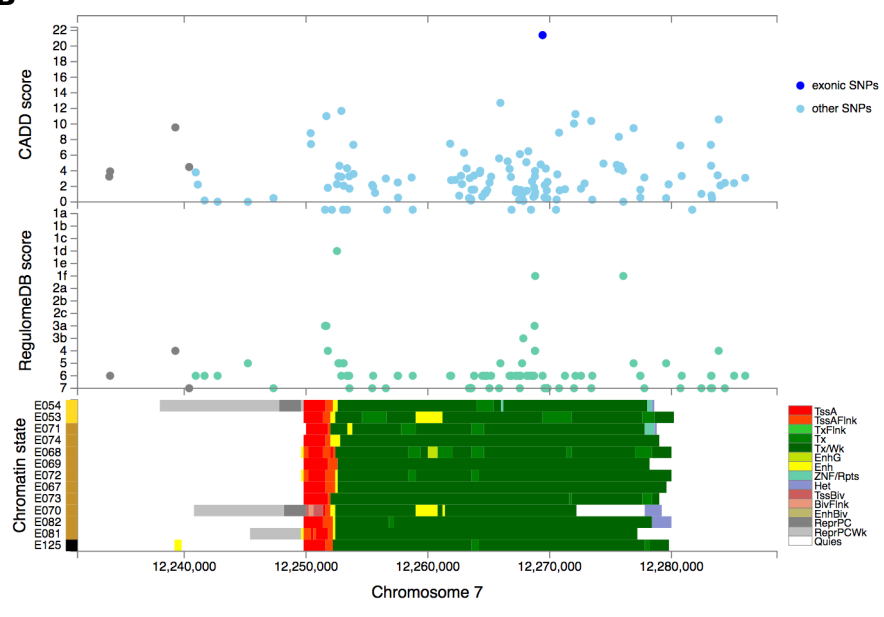

C

Regulome DB

RegulomeDB Categorical Scores

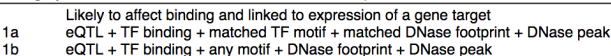

co eQTL + TF binding + matched TF motif + DNase peak

eQTL + TF binding + matched TF motif
eQTL + TF bindinglinase peak

Likely to affect binding

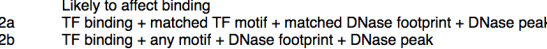

Thinding + mat

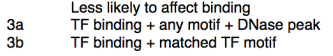

$\begin{array}{ll}4 & \text { Minimal binding evidence } \\ 5 & \text { TF Finding DNase peak } \\ \text { TF binding or DNase peak }\end{array}$

$\begin{array}{lll}5 & \text { TF binding or DNase } \\ 6 & \text { Motif hit } \\ 7 & \end{array}$

No binding eviden
No evidence
he

7.

"External link to RegulomeDB from SNP table (when one of the SNPs is clicked) will open a new tab. rsID does not always match sinco Regula

15-core chromatin state

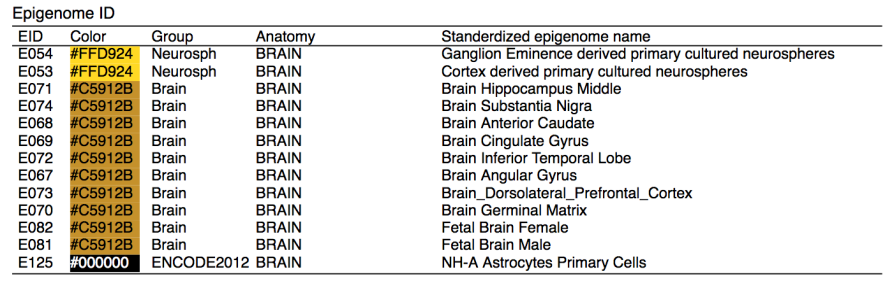

When 15-core chromatin state is included in the plot and $>30$ cell types are selected, the labels of $Y$-axis are omitted. The order of the as the legend table.

Figure 8 Variant rs1990621 functional annotation and local plot. A) Local plot showed the zoom-in view of the hit in chromosome 7 with the top lead SNP rs 1990621 labeled with dark purple. Nearby SNPs were also mainly located in the TMEM106B gene region and color coded with LD r2 thresholds. B) Bottom panel showed combined CADD score, RegulomeDB score, and Chromatin state of the region shown in the top panel. C) Regulome DB and chromatin state explanation. 

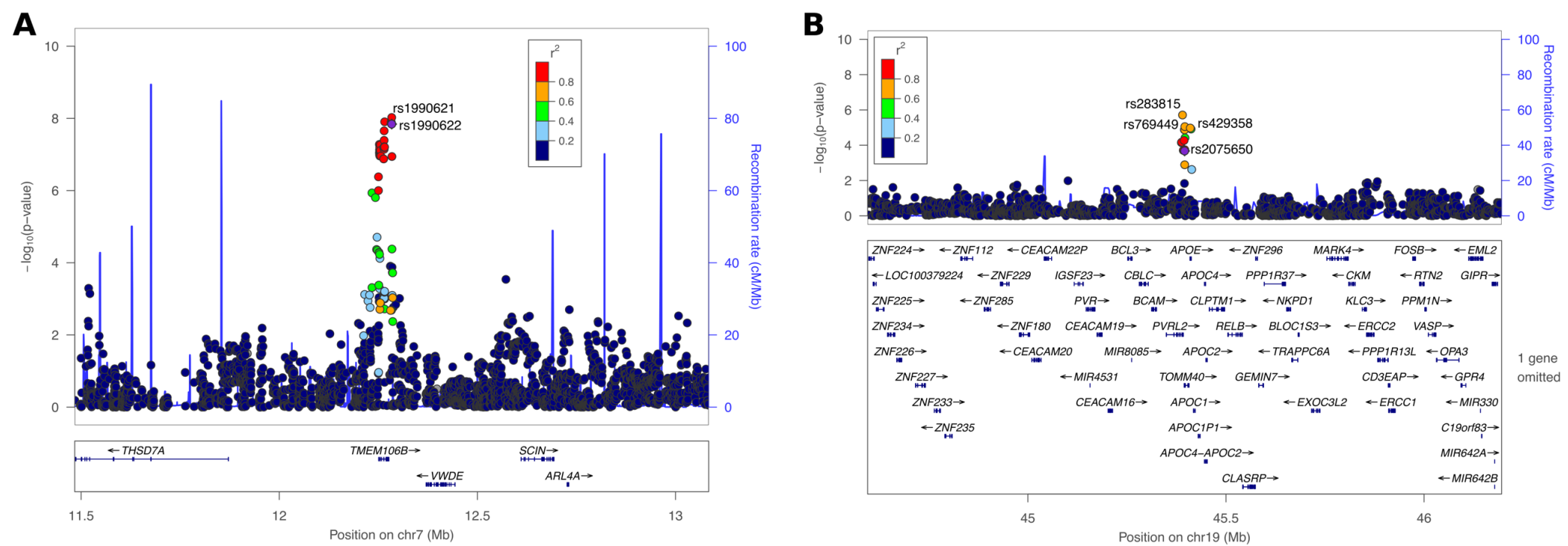

Figure 9 TMEM106B and $A P O E$ regions local plot. A) Local plot showed the zoom-in view of the hit in chromosome 7 with target SNP rs 1990622 labeled with dark purple, and the top leading SNP is rs1990621. Nearby SNPs were also mainly located in the TMEM106B gene region and color coded with LD r2 thresholds. B) Local plot showed the zoom-in view of the hit in chromosome 19 with target SNP rs2075650 labeled with dark purple, and the top three leading SNPs are rs283815, rs769449, and rs429358. Nearby SNPs were also mainly located in the TOMM40/APOE gene region and color coded with LD r2 thresholds. One gene omitted in this region is SNRPD2. 